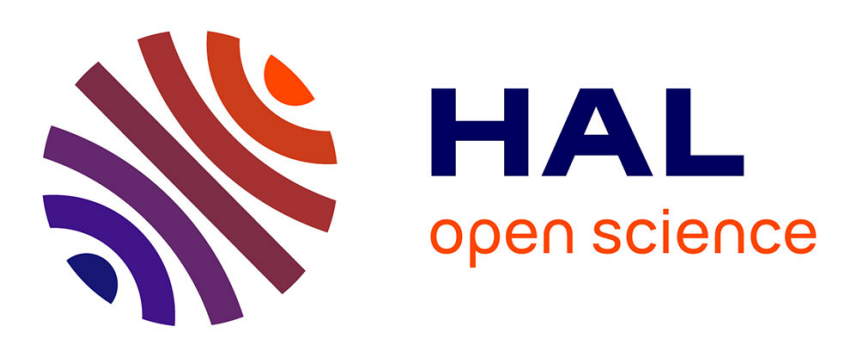

\title{
Regularity and Lyapunov stabilization of weak entropy solutions to scalar conservation laws
}

Sébastien Blandin, Xavier Litrico, Maria Laura Delle Monache, Benedetto Piccoli, Alexandre Bayen

\section{- To cite this version:}

Sébastien Blandin, Xavier Litrico, Maria Laura Delle Monache, Benedetto Piccoli, Alexandre Bayen. Regularity and Lyapunov stabilization of weak entropy solutions to scalar conservation laws. IEEE Transactions on Automatic Control, 2017, 62 (4), pp.1620-1635. hal-01267722

\section{HAL Id: hal-01267722 \\ https://inria.hal.science/hal-01267722}

Submitted on 4 Feb 2016

HAL is a multi-disciplinary open access archive for the deposit and dissemination of scientific research documents, whether they are published or not. The documents may come from teaching and research institutions in France or abroad, or from public or private research centers.
L'archive ouverte pluridisciplinaire HAL, est destinée au dépôt et à la diffusion de documents scientifiques de niveau recherche, publiés ou non, émanant des établissements d'enseignement et de recherche français ou étrangers, des laboratoires publics ou privés. 


\title{
Regularity and Lyapunov stabilization of weak entropy solutions to scalar conservation laws
}

\author{
Sébastien Blandin, Xavier Litricoł Maria Laura Delle Monache‡ \\ Benedetto Piccoli§, Alexandre Bayen
}

\begin{abstract}
We consider the problem of Lyapunov boundary stabilization of the weak entropy solution to a scalar conservation law with strictly convex flux in one dimension of space, around a uniform equilibrium. We show that for a specific class of boundary conditions, the solution to the initial-boundary value problem for an initial condition with bounded variations can be approximated arbitrarily closely in the $L^{1}$ norm by a piecewise smooth solution with finitely many discontinuities. The constructive method we present designs explicit boundary conditions in this class, which guarantee Lyapunov stability of the weak entropy solution to the initial-boundary value problem. We show how the greedy control, obtained by maximizing the decrease of the natural Lyapunov function, may fail to asymptotically stabilize and a brute force control generates unbounded variation of traces. We then design a stabilizing control, which avoid oscillations, and propose a nonlocal technique (depending on time and the whole initial datum) which optimizes the convergence time. Controllers performance is illustrated on numerical benchmarks using the Godunov scheme.
\end{abstract}

\section{Introduction}

\subsection{Motivation}

The conservation principle is one of the most fundamental modeling principles for physical systems. Statements of conservation of mass, momentum, energy are at the center of modern classical physics. For distributed dynamical systems, this principle can be written in conservation law form with the use of partial

\footnotetext{
* Sébastien Blandin is a Research Scientist, IBM Research, Singapore (email: sblandin@sg.ibm.com). Corresponding author.

${ }^{\dagger}$ Xavier Litrico is Scientific Director at Suez Water France, Director of LyRE, Suez R\&D Center, Bordeaux, France (xavier.litrico@lyonnaise-des-eaux.fr).

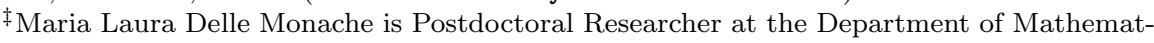
ical Sciences, Rutgers University, Camden (ml.dellemonache@rutgers.edu).

$\S$ Benedetto Piccoli is the Joseph and Loretta Lopez Chair Professor of Mathematics, Department of Mathematical Sciences, and Director, Center for Computational and Integrative Biology, Rutgers University, Camden (piccoli@camden.rutgers.edu).

"Alexandre Bayen is the Chancellor's Professor, Department of Electrical Engineering and Computer Sciences, Department of Civil and Environmental Engineering, University of California, Berkeley (e-mail: bayen@berkeley.edu).
} 
differential equations (PDE). The problem of well-posedness of the partial differential equation is concerned with the existence, uniqueness, and continuous dependence of the solution to the problem data [35].

First existence results for scalar conservation laws in one dimension of space date back to [63]. For hyperbolic systems of conservation laws in one dimension of space, existence results were provided in [41] with the introduction of the random choice method. Existence and uniqueness in the scalar case for several dimensions of spaces were proven in [51], and to this date constitute the only general results known on well-posedness for several dimensions of space. Existence and uniqueness for $n \times n$ hyperbolic systems of conservation laws in one-dimension of space was shown only recently, see $[21,23]$ for systems with genuinely nonlinear or linearly degenerate characteristic families, and $[9,15]$ for the general case of systems of strictly hyperbolic conservation laws. The global well-posedness of solutions to hyperbolic systems of conservation laws in several dimensions of space is still largely open.

The keystone of well-posedness results, and a standard argument for constructive existence proofs, is the consideration of a functional space in which small variations in the problem data, i.e. initial condition for the Cauchy problem, initial and boundary conditions for the initial-boundary value problem (IBVP), create only small variations in the tentative solution.

The control problem is posed from a different perspective, and in different terms. In the case of boundary control [50], the control problem consists of an objective trajectory for the system in a given functional space, and the knowledge of an initial condition. The problem of control [26] or stabilization [49] consists in the existence and design of a controller, i.e. boundary conditions in the case of boundary control or stabilization, for which the solution to the partial differential equation stays in a domain prescribed by the objective trajectory.

In this article we propose to show Lyapunov stability of the solution to the initial-boundary value problem associated with a scalar conservation law under suitably designed boundary conditions. We consider a scalar conservation law with smooth strictly concave or convex flux in one dimension of space. This partial differential equation is called the Burgers equation [45] in the case of a quadratic convex flux, and in the case of a concave flux, corresponds to the Lighthill-Whitham-Richards (LWR) [58, 69] PDE in its various forms, used in particular for macroscopic traffic flow modeling. The problem is defined in the following section.

\subsection{Problem statement}

Consider the scalar conservation law in one dimension of space

$$
\partial_{t} u+\partial_{x} f(u)=0
$$

on the domain $\Omega \doteq\{(t, x) \mid t \geq 0$ and $a \leq x \leq b\}$. The flux function $f(\cdot)$ is assumed to be smooth (infinitely differentiable) and strictly convex or strictly concave $^{1}$. The initial-boundary value problem (IBVP) for (1) in $\Omega$ with initial

\footnotetext{
${ }^{1}$ this is equivalent to the condition of genuine nonlinearity of the characteristic field.
} 
condition $u_{0}:(a, b) \mapsto \mathbb{R}$, and boundary conditions $u_{a}, u_{b}: \mathbb{R}^{+} \mapsto \mathbb{R}$, reads

$$
\begin{aligned}
& \partial_{t} u+\partial_{x} f(u)=0 \\
& u(0, x)=u_{0}(x) \\
& u(t, a)=u_{a}(t), u(t, b)=u_{b}(t) .
\end{aligned}
$$

The Lyapunov boundary stabilization problem can now be formulated.

Definition 1 Given a stationary solution $u^{*}$ to the PDE (1), the Lyapunov (resp. asymptotic) boundary stabilization problem consists in the existence of boundary conditions $u_{a}, u_{b}$ depending on initial condition $u_{0}$ with bounded variations (see section 2.1) such that the following is true. The IBVP (2)-(3)-(4) is well-posed and its solution is (resp. asymptotically) stable in the sense of Lyapunov at $u^{*}$.

Note that Lyapunov (resp. asymptotic) stabilization consists in the existence of a positive definite function decreasing (resp. vanishing) in time along trajectories of the system.

Stationary solutions to the PDE include constant solutions and solutions with a single stationary jump discontinuity, called shock. In this article we address the case of constant solutions.

The well-posedness of the IBVP (2)-(3)-(4) is critical to the definition of the problem, since the design of arbitrary boundary conditions can make the problem ill-posed (see [74] for an illustration on the LWR equation in the case of traffic). This would lead to a discrepancy between the control implemented and its realized value in the system, and a divergence between the desired trajectory of the system and its real trajectory. In the case of traffic, it corresponds for instance to installing a green traffic light at the location of a traffic jam with the intended goal that cars in the jam adopt the corresponding free-flow speed. For well-posedness of the IBVP (2)-(3)-(4), the PDE (1) and the boundary conditions (4) must be understood in the weak sense. The weak formulation is presented in Section 2.2.

The following section consists in a review of results on boundary control of partial differential equations.

\subsection{Literature review}

The problem of boundary control of partial differential equations has been the focus of ongoing research. A large volume of literature is concerned with the problem of boundary control of the viscous Burgers equation. Control results can be found in $[24,25,28,39,40,47,48,53,62,65,72]$. A Lyapunov approach, from which the method presented in this article was inspired, has been proposed in [48], for classical solutions to the PDE.

Lyapunov methods for classical solutions of networks of scalar conservation laws have also been proposed in [13, 67], and for classical solutions of $2 \times 2$ systems with characteristics speeds of constant opposite sign in [27, 33]. Similar work on boundary damping techniques with applications to the Saint-Venant equations has been proposed in $[30,68]$. Switching techniques for linear hyperbolic systems are investigated in [4], and asymptotic stabilization using a stationary feedback law in [66]. A frequency domain framework [59] has been used to design a boundary control for the linearized Saint-Venant equations in [60] 
(see also [44] for boundary control of the Saint-Venant equations). A specific method for flat systems has been introduced in [36]. For a recent application of backstepping, the reader is referred to [76].

Methods developed specifically for well-posedness results of conservation laws have also been applied to the problem of boundary control. In [6], a wavefront tracking method was used to compute the fixed horizon attainable set of initial-boundary value problem solutions of Temple systems of conservation laws. In $[7,5]$, the authors studied the attainable sets for the same problem, i.e. the set of functions which can be reached by acting with controls. The problem of asymptotic stabilization by boundary controls was studied also in $[20,8]$ under the non-characteristic condition. In particular, the paper [20] provides a counterexample to exact controllability.

Frameworks arising from the field of optimal control have also been applied to this problem [46]. In [10], the authors proposed a viability framework for a Hamilton- Jacobi equation corresponding to an integral form of the Burgers equation, which leads to lower semi-continuous solutions. A linear relaxation method for the nonlinear discrete dynamics of the asymmetric cell-transmission model, a specific instantiation of the Godunov scheme for the LWR equation, has also been proposed in [43], with subsequent global optimization of the obtained equivalent convex problem.

One of the challenging features of conservation laws is the apparition of discontinuities in finite time in the solution to the Cauchy problem, even for smooth initial condition. This yields difficulties for most control approaches since classical control methods are not well-suited to handle discontinuities. In the case of parametric initial conditions, the problem of differentiating the solution to the scalar conservation law with respect to the initial condition parameter has been specifically addressed in [12] from a mathematical perspective. One must also note the more general shift differentiability method for functionals on $L^{1} \cap B V$ introduced in $[14,22]$ (see section 2.1 for a definition of the class of $B V$ functions).

A second specific challenge of boundary control of the solution to a conservation law, also due to the non-linearity of the PDE, is the fact that weak boundary conditions have to be considered. In this work, we specifically account for these two issues and show the stability of the weak entropy solution to the scalar conservation law (1). The main contributions of this article are the following:

- Well-posedness result: we consider data with a finite number of shocks and otherwise positive gradient and show well-posedness of the IBVP associated to a stabilizing control with the scalar conservation law in this class. The corresponding piecewise smooth solution can approximate arbitrarily well the solution to an IBVP with $B V$ initial condition.

- Stabilization of a Lyapunov function candidate: we show that there exists a strictly decreasing Lyapunov function for the scalar conservation law for appropriate boundary conditions.

- Controller design: we identify explicitly the boundary conditions that maximize the decrease rate of the Lyapunov function and show that they may not stabilize the system. A stabilizing control is then designed. The latter keeps the solution in the piecewise smooth class, can be extended to BV 
data and guarantees asymptotic stability (which in turn implies $L^{2}$ convergence of solutions). We also design nonlocal strategies to improve the convergence time.

The remainder of the article is organized as follows. Section 2 defines the notations used later in the article and states useful lemmas. Section 3 contains the proof of well-posedness of the initial-boundary value problem with piecewisesmooth increasing datum with negative gradient concentrated at a finite number of locations. Preparatory derivations involving the Lyapunov function candidate can be found in Section 4. In Section 5, we show stability of the Lyapunov function for weak entropy solutions to the scalar conservation law remaining in the special class with a finite number of shocks. In Section 6 we design a controller that maximizes the instantaneous decrease of the Lyapunov function identified previously, but highlight several configurations in which asymptotic stability is not achieved by this controller. We also illustrate how a brute force controller introduces unbounded oscillations, in the form of infinite variation of the solution traces. In Section 7 we design a new controller which guarantees asymptotic stability and the existence of solution to the IBVP in the special class of solutions with a finite number of shocks. Moreover, we design nonlocal controllers, depending on the whole initial datum, which improve the convergence time. Numerical examples are proposed in Section 8 , and promising research avenues related to this work in Section 9.

\section{Preliminaries}

In this section, we introduce results subsequently used throughout the article.

\subsection{BV functions}

Consider an interval $J \subset \mathbb{R}$, and a map $u: J \mapsto \mathbb{R}$. The total variation of $u$ is defined as

$$
\text { Tot. Var. }\{u\} \doteq \sup \left\{\sum_{i=1}^{N}\left|u\left(x_{j}\right)-u\left(x_{j-1}\right)\right|\right\}
$$

where the supremum is taken over all $N \geq 1$ and all $(N+1)$-tuples of points $x_{j} \in J$ such that $x_{0}<x_{1}<\ldots<x_{N}$. If the total variation of $u$ is finite then we write $u \in B V$. Specific properties of $B V$ functions leveraged in the following sections are presented below ${ }^{2}$.

Lemma 1 [19] Let $u:(a, b) \mapsto \mathbb{R}^{n}$ have bounded variation. Then for every $x \in(a, b)$, the left and right limits

$$
u(x-) \doteq \lim _{y \mapsto x-} u(y), \quad u(x+) \doteq \lim _{y \mapsto x+} u(y)
$$

are well defined. Moreover, $u$ has at most countably many points of discontinuity.

The following lemma concerns piecewise constant approximability of $B V$ functions.

\footnotetext{
${ }^{2}$ Proofs of these properties can be found in section 2.4 of [19].
} 
Lemma 2 [19] Let $u: \mathbb{R} \mapsto \mathbb{R}^{n}$ be right continuous with bounded variation. Then, for every $\epsilon>0$, there exists a piecewise constant function $v$ such that:

Tot. Var. $\{v\} \leq$ Tot. Var. $\{u\}, \quad\|v-u\|_{L^{\infty}} \leq \epsilon$.

If, in addition,

$$
\int_{-\infty}^{0}|u(x)-u(-\infty)| d x+\int_{0}^{+\infty}|u(x)-u(\infty)| d x<\infty,
$$

then one can find $v$ with the additional property

$$
\|u-v\|_{L^{1}} \leq \epsilon
$$

The space of BV functions and its closure in $L^{1}$ are at the center of wellposedness results for conservation laws using wavefront-tracking methods.

\subsection{Weak solutions to the initial-boundary value problem}

It is well-known that jump discontinuities can arise in finite time in solutions to conservation laws [35]. Thus classical solutions do not exist in general, and it is necessary to consider a more general formulation of the conservation law.

\subsubsection{Weak entropy solution to the Cauchy problem}

The weak formulation of the conservation law is obtained by considering derivatives in the sense of distribution.

Definition 2 A function $u:[0, T] \times \mathbb{R} \mapsto \mathbb{R}$ is a weak solution to the Cauchy problem (2)-(3) if for any continuously differentiable function $\phi$ with compact support contained in $(-\infty, T) \times \mathbb{R}$,

$$
\int_{0}^{T} \int_{-\infty}^{\infty}\left(u \phi_{t}+f(u) \phi_{x}\right) d x d t+\int_{-\infty}^{\infty} u_{0}(x) \phi(0, x) d x=0
$$

and $t \mapsto u(t, \cdot)$ is continuous from $[0, T]$ into $L_{l o c}^{1}$.

Given that $u$ is smooth around a jump discontinuity, integrating the weak formulation (6) yields the Rankine-Hugoniot relation [35] defining the speed $\sigma$ of propagation of jump discontinuities

$$
\sigma \Delta u=\Delta f(u)
$$

where $\Delta u=u_{r}-u_{l}$ is the jump in $u$, with $u_{r}$, respectively $u_{l}$, the value of the right, respectively left, limit of $u$ at the jump location.

To isolate a unique weak solution to a Cauchy problem associated with the conservation law, an additional admissibility condition (see Section 4.5 of [29]) is required. Different conditions have been proposed in the literature. In the scalar case, one of the first admissibility conditions, due to Oleinik [63], states that for a shock joining a left state $u_{l}$ and a right state $u_{r}$, the following inequality must be satisfied for all $u$ between $u_{l}$ and $u_{r}$ :

$$
\frac{f(u)-f\left(u_{l}\right)}{u-u_{l}} \geq \sigma \geq \frac{f(u)-f\left(u_{r}\right)}{u-u_{r}},
$$


where $\sigma$ is the Rankine-Hugoniot speed (7). Kruzkhov [51] showed that it was sufficient to satisfy the entropy inequality condition for a specific family of entropy-entropy flux pairs in the scalar case, yielding the Kruzkhov entropy condition. The Lax admissibility condition [52], which exhibits a convenient geometric interpretation, states that for a shock joining a left state $u_{l}$ and a right state $u_{r}$, the following inequality must be satisfied:

$$
\lambda\left(u_{l}\right) \geq \sigma \geq \lambda\left(u_{r}\right)
$$

where $\lambda(u)$ is the characteristic speed of $u$ (i.e. $f^{\prime}(u)$ ), and $\sigma$ is the RankineHugoniot speed (7). For the case of systems one requires that condition (9) holds for a genuinely nonlinear or linearly degenerate $i$-th characteristic family with $\lambda$ replaced by the $i$-th eigenvalue $\lambda_{i}$ of the Jacobian matrix $D F(u)$. In the scalar case, for a convex flux, these formulations have been proven to be equivalent (see Section 2.1 of [55]). The Lax admissibility condition allows the selection of a particular weak solution.

Definition 3 A function $u:[0, T] \times \mathbb{R} \mapsto \mathbb{R}$ is the weak entropy solution to the Cauchy problem (2)-(3) if it is a weak solution (definition 2), that satisfies the Lax admissibility condition (9).

The definition of weak conditions to the IBVP requires a corresponding statement of weak boundary conditions, presented in the following section.

\subsubsection{Weak boundary conditions}

The first statement of weak boundary conditions was introduced in [11] in the scalar case in multiple dimensions of space, with $C^{2}$ flux and $C^{2}$ initial and boundary datum, using a vanishing viscosity method. In one dimension, this formulation reads:

$$
\begin{aligned}
& \max _{k \in[\alpha, \beta]} \operatorname{sgn}\left(u(t, a)-u_{a}(t)\right)(f(u(t, a))-f(k))=0 \\
& \min _{k \in[\gamma, \delta]} \operatorname{sgn}\left(u(t, b)-u_{b}(t)\right)(f(u(t, b))-f(k))=0
\end{aligned}
$$

for almost all $t>0$, and where $\alpha=\min \left(u(t, a), u_{a}(t)\right), \beta=\max \left(u(t, a), u_{a}(t)\right)$, $\gamma=\min \left(u(t, b), u_{b}(t)\right), \delta=\max \left(u(t, b), u_{b}(t)\right)$, and sgn denotes the sign function. For the case of systems of conservation laws, the interested reader is referred to [16],[71]. In the scalar case, at a left boundary $a$, the corresponding statement of weak boundary conditions derived from the structure of the solution to the Riemann problem is the following.

Definition 4 A function $u: \Omega \mapsto \mathbb{R}$ satisfies the boundary condition $u_{a}$ at a if for almost every time $t$ (in the sense of Lebesgue measure), the solution to the Riemann problem centered at a with initial data

$$
\begin{cases}u_{a}(t) & \text { if } \quad x<a \\ u(t, a) & \text { if } \quad x>a\end{cases}
$$

either does not contain any wave (when left and right initial states are the same), or contains waves with non positive speeds (a wave with zero speed is allowed). Notice that for weak solutions we consider the condition will hold for all times except a finite number. 
The domain of the couples boundary condition-boundary trace satisfying the weak boundary conditions (definition 4) is represented in figure 1 for a Burgers flux. In [54], a simplified formulation is proposed for the scalar case with strictly

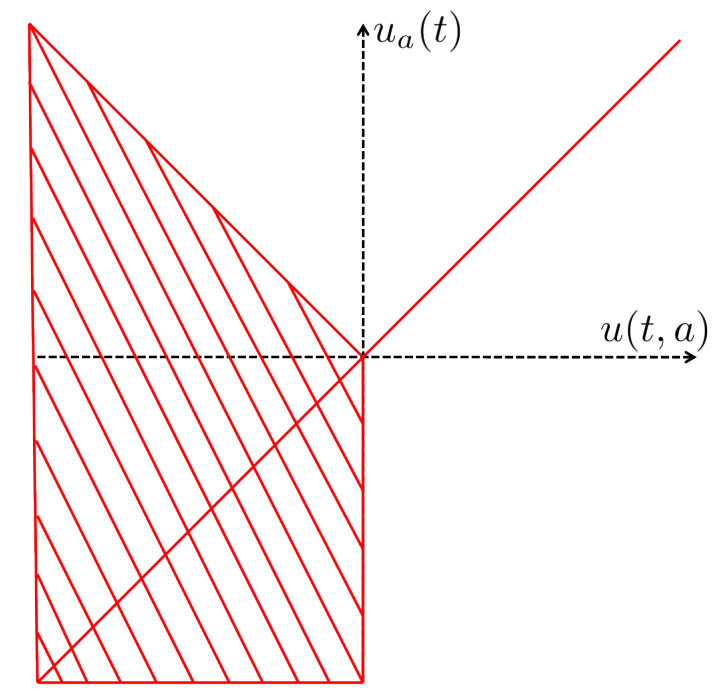

Figure 1: Weak boundary conditions: in the case of a quadratic convex flux centered at 0 . The solution to the Riemann problem with initial datum in the striped domain exhibits a wave with negative speed. For initial datum on the first bisector, no wave arises. The white zone exists only for a zero time duration.

convex continuously differentiable flux functions (see also [37]). Similar formulations were derived for a scalar traffic model in [74] and scalar traffic models on networks with application to estimation in [77], following [38]. In the case of a strictly convex continuously differentiable flux, this statement states that for almost all times $t$, one of the following mutually exclusive conditions must be satisfied:

$$
\left\{\begin{array}{l}
u(t, a)=u_{a}(t) \\
f^{\prime}(u(t, a)) \leq 0 \text { and } f^{\prime}\left(u_{a}(t)\right) \leq 0 \text { and } u(t, a) \neq u_{a}(t) \\
f^{\prime}(u(t, a)) \leq 0 \text { and } f^{\prime}\left(u_{a}(t)\right)>0 \text { and } f(u(t, a)) \geq f\left(u_{a}(t)\right) .
\end{array}\right.
$$

The same can be done at the downstream boundary $x=b$ :

$$
\left\{\begin{array}{l}
u(t, b)=u_{b}(t) \\
f^{\prime}(u(t, b)) \geq 0 \text { and } f^{\prime}\left(u_{b}(t)\right) \geq 0 \text { and } u(t, b) \neq u_{b}(t) \\
f^{\prime}(u(t, b)) \geq 0 \text { and } f^{\prime}\left(u_{b}(t)\right)<0 \text { and } f(u(t, b)) \geq f\left(u_{b}(t)\right) .
\end{array}\right.
$$

A well-posedness result for the IBVP associated with a scalar conservation law (1) with the boundary statement from definition 4 is presented in the following section. 


\subsection{Well-posedness of the initial-boundary value problem}

In [11], a solution satisfying (6) in the scalar case is constructed using a vanishing viscosity method for the weak boundary conditions statement (10)-(11) and is shown to be the admissible solution according to Kruzkhov entropy condition [51].

More recently, an existence result for $n \times n$ systems using wavefront tracking was proposed in [1]. The standard Riemann semigroup (SRS) method, introduced in [18] for the Cauchy problem associated with a Temple system [75] of conservation laws, was extended to the IBVP in $[2,3]$, with the boundary conditions statement from definition 4. In [3], it is shown for a $n \times n$ system that if the SRS exist, its trajectories coincide with wavefront tracking solutions. Uniqueness and continuous dependence is obtained for the case of non-characteristic conditions, and uniqueness for the characteristic case. The SRS is constructed for $2 \times 2$ system in [2], and for the case of $n \times n$ system in [32]. The stability of the IBVP with two boundaries was established via vanishing viscosity for $2 \times 2$ systems in [73]. We state in the scalar case for a static boundary the main result of [2] for characteristic boundary conditions, obtained for $2 \times 2$ systems with continuous boundary (see theorem $C$ of [2]). A general result for $n \times n$ systems was established in [8].

Theorem 1 [2] Let $f$ be a smooth map such that the equation (1) is strictly hyperbolic with characteristic field linearly degenerate or genuinely nonlinear (i.e. $f$ is linear, convex or concave). For every $\delta>0$ there exists $L>0$ and a continuous semigroup $S$ defined for data in $L^{1} \cap B V$ with total variation bounded by $\delta$, such that

- The map $t \mapsto u(t, \cdot)$ yields a weak solution to the IBVP (2)-(3)-(4).

- For piecewise constant initial and boundary data, the trajectories of the semigroup coincide with the solution to the IBVP obtained by piecing together the standard solutions to the Riemann problems at the points of discontinuity of the initial condition and at the boundary.

- For initial data $u_{0}^{\prime}, u_{0}^{\prime \prime}$, boundary data $u_{a}^{\prime}, u_{a}^{\prime \prime}, u_{b}^{\prime}, u_{b}^{\prime \prime}$ in $L^{1} \cap B V$ with total variation bounded by $\delta$, let $u^{\prime}, u^{\prime \prime}$ denote the corresponding trajectories of the semigroup $S$, and $t^{\prime}, t^{\prime \prime}>0$, then

$$
\begin{array}{r}
\left\|u^{\prime}\left(t^{\prime}, \cdot\right)-u^{\prime \prime}\left(t^{\prime \prime}, \cdot\right)\right\|_{1} \leq L\left(\left|t^{\prime}-t^{\prime \prime}\right|+\left\|u_{0}^{\prime}-u_{0}^{\prime \prime}\right\|_{1}+\left\|u_{a}^{\prime}-u_{a}^{\prime \prime}\right\|_{1}\right. \\
\left.+\left\|u_{b}^{\prime}-u_{b}^{\prime \prime}\right\|_{1}\right) .
\end{array}
$$

We also refer the interested reader to the work of Otto [64] for the case where the entropy solution does not have traces at the boundary, see also [70]. In the following section we use this result in the case of a left and a right boundary to show that we can restrict our Lyapunov analysis to the case of piecewise smooth data.

\section{Approximation of solution to initial-boundary value problem by piecewise smooth solution}

In this section, we present results on the approximability of the solution to an IBVP with initial condition in BV by the solution to an IBVP with piecewise 
smooth solution at all times. We show that the solution to the IBVP with BV data can be approximated arbitrarily closely in the $L^{1}$ norm by the solution to an IBVP with piecewise smooth data. We define the required regularity class used throughout the article.

Definition 5 We note $P W S^{+}$the class of piecewise smooth functions $f: \mathbb{R} \mapsto$ $\mathbb{R}$ such that

- $f^{\prime}$ is positive (where defined).

- $f$ has only downward jumps (i.e. $f^{\prime}$ seen as measure has only negative Dirac masses).

We now state the approximability result.

Theorem 2 Consider $T>0, a<b$, and let $u_{0}:(a, b) \mapsto \mathbb{R}, u_{a}, u_{b}:(0, T) \mapsto \mathbb{R}$ be functions with bounded total variation. For every $\epsilon>0$, there exists $u_{0}^{\epsilon}$ : $(a, b) \mapsto \mathbb{R}$ in $P W S^{+}$and piecewise constant boundary data $u_{a}^{\epsilon}, u_{b}^{\epsilon}:(0, T) \mapsto \mathbb{R}$ such that

$$
\left\|u_{a}(t)-u_{a}^{\epsilon}(t)\right\|_{1} \leq \epsilon, \quad\left\|u_{b}(t)-u_{b}^{\epsilon}(t)\right\|_{1} \leq \epsilon
$$

and the solution $u$ to the IBVP for equation (1) and data $\left(u_{0}, u_{a}, u_{b}\right)$ and the solution $u^{\epsilon}$ to the IBVP for equation (1) and data $\left(u_{0}^{\epsilon}, u_{a}^{\epsilon}, u_{b}^{\epsilon}\right)$ satisfy:

$$
\forall 0 \leq t \leq T, \quad\left\|u(t, \cdot)-u^{\epsilon}(t, \cdot)\right\|_{1} \leq \epsilon
$$

Proof 1 Using lemma 2 in the compact domain $[a, b]$, we can approximate the initial condition condition $u_{0}$ arbitrarily closely in the $L^{1}$ sense by a piecewise constant function $u_{0}^{\epsilon}$ with a finite number of discontinuities and lower total variation. Also we can replace the upward jumps of $u_{0}^{\epsilon}$ with smooth increasing functions without increasing the total variation. We can also approximate boundary data by piecewise constant functions of lower variation.

Since $u_{0}^{\epsilon}$ has only positive derivative, no new shock can form in the solution inside the domain. Moreover, a finite number of shocks will be introduced by the boundary conditions. Therefore the solution $u^{\epsilon}$ will remain in the class $P W S^{+}$.

Using the continuous dependence result of Theorem 1, the resulting trajectories $u, u_{\epsilon}$ of the semigroup can be made arbitrarily close in the $L^{1}$ norm by controlling the distance between the initial conditions.

We show that under suitable boundary conditions, the solution to the IBVP with piecewise smooth data is piecewise smooth.

Theorem 3 Let $T, \delta>0, a<b$, and let $u_{0}:(a, b) \mapsto \mathbb{R}$ be in PWS and $u_{a}, u_{b}:(0, T) \mapsto \mathbb{R}$ be piecewise constant. Let $u$ denote the solution to the IBVP (6)-(3)-(12). At all times $0 \leq t \leq T, u(t, \cdot)$ is piecewise smooth.

Proof 2 Let $x_{1}, \ldots, x_{N}$ denote the locations of the discontinuities in the initial condition, including $a, b$. We construct the solution to the IBVP by piecing together the shock waves created at $x_{i}$, and the classical solution constructed by the method of characteristics between the waves. By definition, the solution created is a solution in the sense of (6) since equation (1) is satisfied in the classical sense between the waves, and the shock waves propagate according to the Rankine-Hugoniot relation (7). It is also the admissible solution according to 
the Lax condition (9) thus the constructed solution coincides with the trajectory of the semigroup from theorem 2 (see [21]).

We now show that at all time, the number of discontinuities is finite. Since there is a finite number of discontinuities in the initial and boundary conditions, a finite number of discontinuities enters the space-time domain. In the scalar case the interaction of two discontinuities creates no more than one discontinuity, so the interaction of discontinuities contributes to decreasing their number. Finally as mentioned above, since the initial and boundary conditions are piecewise smooth increasing, all discontinuities are created at time 0 or at the boundary.

In the next section, we present the Lyapunov stability analysis for functions in $\mathrm{PWS}^{+}$.

\section{Lyapunov analysis}

In this section, we propose a Lyapunov function and compute its derivative. In the following we call $\tilde{u}=u-u^{*}$ where $u^{*}$ is a constant, hence stationary, solution around which we want to stabilize the system, and $u$ is the solution to the IBVP associated with the scalar conservation law (1). Following the results from section 3, we assume that $u$ is in $\mathrm{PWS}^{+}$.

\subsection{Lyapunov function candidate}

We consider the classical Lyapunov function candidate [48, 50]:

$$
V(t)=\frac{1}{2} \int_{a}^{b} \tilde{u}^{2}(t, x) d x=\frac{1}{2} \int_{a}^{b}\left(u(t, x)-u^{*}\right)^{2} d x,
$$

where $u$ is a weak solution to the scalar conservation law. From definition 3 , we have $t \mapsto u(t, \cdot)$ continuous from $[0, T]$ to $L^{1}$, and the function $V(\cdot)$ is well-defined and continuous. We index the jump discontinuities of $u(t, \cdot)$ in increasing order of their location at time $t$ by $i=0, \ldots, N(t)$, including for notational purposes the boundaries $a, b$, with $x_{0}(t)=a$ and $x_{N}(t)=b$. The Lyapunov function candidate can be rewritten as:

$$
V(t)=\frac{1}{2} \sum_{i=0}^{N(t)-1} \int_{x_{i}(t)}^{x_{i+1}(t)} \tilde{u}^{2}(t, x) d x .
$$

From Theorem 3, we know that for all integer $i \in[0, N(t))$, the function $u(t, \cdot)$ is smooth in the domain $\left(x_{i}(t), x_{i+1}(t)\right)$, thus $\partial_{t} u(t, \cdot)$ exists and is continuous for $t$ such that $x_{i}(t)<x_{i+1}(t)$. Since discontinuity trajectories are differentiable with speed given by the Rankine-Hugoniot relation (7), it follows that at any time $t$ such that $N(t)$ is constant in a neighborhood of $t$ and the boundary trace is continuous, the function $V(\cdot)$ is differentiable.

\subsection{Differentiation of the Lyapunov function candidate}

In this section, we compute the derivative of the Lyapunov function candidate (15), at any time $t$ such that $N(t)$ is constant in a neighborhood and the 
boundary trace is continuous. Differentiating expression (16) yields:

$$
\begin{aligned}
\frac{d V}{d t}(t)= & \frac{1}{2} \sum_{i=0}^{N(t)-1} \int_{x_{i}(t)}^{x_{i+1}(t)} \partial_{t} \tilde{u}^{2} d x \\
& +\frac{1}{2} \sum_{i=0}^{N(t)-1}\left[\tilde{u}^{2}\left(t, x_{i+1}(t)-\right) \frac{d x_{i+1}}{d t}(t)-\tilde{u}^{2}\left(t, x_{i}(t)+\right) \frac{d x_{i}}{d t}(t)\right] .
\end{aligned}
$$

As detailed at the end of section 4.1, the term under the sum is smooth, and we can write $\partial_{t} \tilde{u}^{2}=2 \tilde{u} \partial_{t} \tilde{u}$. Since $u$ satisfies the conservation law (1), we have $\partial_{t} \tilde{u}=-\partial_{x} f\left(\tilde{u}+u^{*}\right)$. The derivative of the Lyapunov function can be written as:

$$
\begin{aligned}
& \frac{d V}{d t}(t)=-\sum_{i=0}^{N(t)-1} \int_{x_{i}(t)}^{x_{i+1}(t)} \tilde{u} \partial_{x} f\left(\tilde{u}+u^{*}\right) d x \\
& +\frac{1}{2} \sum_{i=0}^{N(t)-1}\left[\tilde{u}^{2}\left(t, x_{i+1}(t)-\right) \frac{d x_{i+1}}{d t}(t)-\tilde{u}^{2}\left(t, x_{i}(t)+\right) \frac{d x_{i}}{d t}(t)\right] .
\end{aligned}
$$

By integrating by parts the sum terms, we obtain:

$$
\begin{aligned}
& \frac{d V}{d t}(t)=-\sum_{i=0}^{N(t)-1}\left[\tilde{u} f\left(\tilde{u}+u^{*}\right)\right]_{x_{i}(t)}^{x_{i+1}(t)} \\
& +\sum_{i=0}^{N(t)-1} \int_{x_{i}(t)}^{x_{i+1}(t)} f\left(\tilde{u}+u^{*}\right) \partial_{x} \tilde{u} d x \\
& +\frac{1}{2} \sum_{i=0}^{N(t)-1}\left[\tilde{u}^{2}\left(t, x_{i+1}(t)-\right) \frac{d x_{i+1}}{d t}(t)-\tilde{u}^{2}\left(t, x_{i}(t)+\right) \frac{d x_{i}}{d t}(t)\right] .
\end{aligned}
$$

and if we note $F(\cdot)$ a primitive function of the flux function $f(\cdot)$ we have:

$$
\begin{aligned}
& \frac{d V}{d t}(t)=\tilde{u}(t, a) f\left(\tilde{u}(t, a)+u^{*}\right)-\tilde{u}(t, b) f\left(\tilde{u}(t, b)+u^{*}\right) \\
& -F\left(\tilde{u}(t, a)+u^{*}\right)+F\left(\tilde{u}(t, b)+u^{*}\right) \\
& +\sum_{i=1}^{N(t)-1}\left[\Delta_{i}\left(\tilde{u} f\left(\tilde{u}+u^{*}\right)-F\left(\tilde{u}+u^{*}\right)\right)-\frac{1}{2} \frac{d x_{i}}{d t}(t) \Delta_{i} \tilde{u}^{2}\right],
\end{aligned}
$$

where $\Delta_{i}$ is defined around the discontinuity $x_{i}(t)$ as in equation (7). Using the Rankine-Hugoniot relation, defined in equation (7), to write the speed of the jump discontinuity $d x_{i}(t) / d t$ as a function of the left and right jump values we 
obtain:

$$
\begin{gathered}
\frac{d V}{d t}(t)=\tilde{u}(t, a) f\left(\tilde{u}(t, a)+u^{*}\right)-\tilde{u}(t, b) f\left(\tilde{u}(t, b)+u^{*}\right) \\
-F\left(\tilde{u}(t, a)+u^{*}\right)+F\left(\tilde{u}(t, b)+u^{*}\right) \\
+\sum_{i=1}^{N(t)-1} \Delta_{i}\left(\tilde{u} f\left(\tilde{u}+u^{*}\right)-F\left(\tilde{u}+u^{*}\right)\right) \\
-\sum_{i=1}^{N(t)-1} \frac{\tilde{u}\left(t, x_{i}-\right)+\tilde{u}\left(t, x_{i}+\right)}{2} \Delta_{i} f\left(\tilde{u}+u^{*}\right) .
\end{gathered}
$$

In equation (18) we gather the first four terms that depend on the boundary trace of the solution, and the last two terms that depend on the shock dynamics inside the domain. In the following section, we analyze the stability properties of the internal terms.

\subsection{Internal stability}

The last two terms of equation (18) correspond to jump discontinuity in the solution and are neither observable nor controllable from the boundaries. We now show that these terms have a stabilizing effect on the Lyapunov function candidate (15).

Proposition 1 Given a constant solution $u^{*}$ to the scalar conservation law (1), we have the following inequality

$$
\sum_{i=1}^{N(t)-1}\left[\Delta_{i}\left(\tilde{u} f\left(\tilde{u}+u^{*}\right)-F\left(\tilde{u}+u^{*}\right)\right)-\frac{\tilde{u}\left(t, x_{i}-\right)+\tilde{u}\left(t, x_{i}+\right)}{2} \Delta_{i} f\left(\tilde{u}+u^{*}\right)\right] \leq 0
$$

i.e. the jump discontinuity dynamics of the solution $u$ to the IBVP, contributes to the decrease of the Lyapunov function candidate (15).

Proof 3 In order to show that the term (19) is negative, we show that each term in the sum is negative. If we note $u_{l}, u_{r}$ the value of $u$ on the left and on the right of the jump discontinuity, respectively, and $\tilde{u}_{l}, \tilde{u}_{r}$ the corresponding reduced variables, we want to show that

$$
\begin{aligned}
\left(\tilde{u}_{r} f\left(\tilde{u}_{r}+u^{*}\right)-F\left(\tilde{u}_{r}\right.\right. & \left.\left.+u^{*}\right)\right) \\
& -\left(\tilde{u}_{l} f\left(\tilde{u}_{l}+u^{*}\right)-F\left(\tilde{u}_{l}+u^{*}\right)\right) \\
& \quad-\frac{\tilde{u}_{l}+\tilde{u}_{r}}{2}\left(f\left(\tilde{u}_{r}+u^{*}\right)-f\left(\tilde{u}_{l}+u^{*}\right)\right) \leq 0 .
\end{aligned}
$$

Equivalently, in the original state variable $u=\tilde{u}+u^{*}$, we have:

$$
\begin{aligned}
& {\left[\left(\left(u_{r}-u^{*}\right) f\left(u_{r}\right)-F\left(u_{r}\right)\right)-\left(\left(u_{l}-u^{*}\right) f\left(u_{l}\right)-F\left(u_{l}\right)\right)\right]} \\
& \quad-\frac{u_{l}+u_{r}-2 u^{*}}{2}\left(f\left(u_{r}\right)-f\left(u_{l}\right)\right) \leq 0 .
\end{aligned}
$$

This can be rewritten as:

$$
F\left(u_{l}\right)-F\left(u_{r}\right)+\frac{1}{2}\left(u_{r}-u_{l}\right)\left(f\left(u_{r}\right)+f\left(u_{l}\right)\right) \leq 0,
$$


which can be obtained from the Oleinik condition(8), here by integration the left inequality of (8) between $u_{l}$ and $u_{r}$. Thus any solution satisfying the Oleinik entropy condition benefits from stability of the jump discontinuity dynamics.

Remark 1 Given that stability of the jump discontinuity dynamics is implied by the Oleinik entropy condition, since the flux function is strictly convex, we see that the internal dynamics is strictly stabilizing, i.e. we have a strict decrease of the Lyapunov function.

Remark 2 When the number of shocks is constant inside the domain and the trace of the solution is continuous, the internal dynamics is stabilizing in the sense that it contributes to the decay of the Lyapunov function candidate (15). Since the Lyapunov function candidate is continuous, the internal dynamics is always stabilizing. This is critical for boundary stabilization where the control action cannot apply directly inside the domain (unlike the case of distributed control [31, 34, 57]). From equation (20), one can note that the magnitude of the internal stability does not depend on the equilibrium $u^{*}$.

Remark 3 At a time $t$ at which the number of discontinuities is not constant or the boundary trace is not continuous, the Lyapunov function is not differentiable, however the difference between the right and left derivative at $t^{+}$and $t^{-}$, respectively, can be computed. This is addressed in section 6 .

If the trace of the solution to the IBVP always takes the value of the boundary condition, it is clear that the solution is stabilizable using boundary control since it amounts to finding boundary controls $u_{a}, u_{b}$ such that $g\left(u_{a}\right)<g\left(u_{b}\right)$, where $g: x \mapsto\left(x-u_{*}\right) f(x)-F(x)$ is not constant (strict convexity of $f$ ).

However, as described in section 2.2.2, this assumption may render the IBVP ill-posed and thus the control inapplicable. In the following section, we show that the Lyapunov function is stabilizable under the proper statement of weak boundary conditions from definition 4 .

\section{$5 \quad$ Well-posed boundary stability}

In this section, we motivate and define the control space and propose a stabilizing controller.

\subsection{Control space}

Due to the emergence of jump discontinuities in the solution to the conservation law (1), weak solutions have to be considered, and boundary conditions have to be considered in the weak sense. It means that it is not always possible to enforce a specific value of the boundary condition. This leads to considering the domain where the boundary controls apply to the solution of the IBVP (introduced in [17] for the specific case of a Burgers flux).

Definition 6 Let us denote $s_{\min }, s_{\max }$ the minimal and maximal speed of the waves composing the solution to the Riemann problem at the boundary. The control space at the left boundary is the set of pairs $\left(u_{l}, u_{r}\right)$ such that either no wave is generated by the Riemann problem $\left(u_{l}=u_{r}\right)$, or $s_{\min } \geq 0$ and $s_{\max }>0$. The control space at the right boundary is the set of pairs $\left(u_{l}, u_{r}\right)$ 
such that either no wave is generated by the Riemann problem $\left(u_{l}=u_{r}\right)$, or $s_{\min }<0$ and $s_{\max } \leq 0$.

Definition 4 from section 2.2.2 and definition 6 are compatible. Definition 4 is a weak formulation that characterizes the couples boundary condition-boundary trace prevailing for non-zero time durations. Definition 6 categorizes the couples boundary conditions-boundary trace, potentially existing for zero time durations, leading to the boundary trace instantaneously taking the value of the boundary condition (see figure 1 and figure 2).

Proposition 2 Let $m$ denote the minimizer of the strictly convex flux function $f$. The control spaces $\mathcal{C}_{a}, \mathcal{C}_{b}$ at the left and right boundaries, respectively, are characterized as the set of pairs $\left(u_{l}, u_{r}\right)$ such that one of the following properties is satisfied

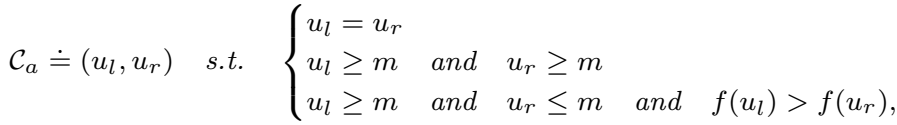

$$
\begin{aligned}
& \mathcal{C}_{b} \doteq\left(u_{l}, u_{r}\right) \quad \text { s.t. }\left\{\begin{array}{l}
u_{l}=u_{r} \\
u_{l} \leq m \quad \text { and } \quad u_{r} \leq m \\
u_{l} \geq m \quad \text { and } \quad u_{r} \leq m \quad \text { and } f\left(u_{l}\right)<f\left(u_{r}\right) .
\end{array}\right.
\end{aligned}
$$

Proof 4 Following definition 6, we characterize the cases in which the solution to the Riemann problem with initial datum $\left(u_{l}, u_{r}\right)$ exhibits no wave (first line of equations (21)-(22)), or waves entering the domain, with at least one noncharacteristic wave speed. The second line of equations (21)-(22) corresponds to entering shock waves or rarefaction waves arising between left and right states with characteristic speed of the same sign, and the third line corresponds to the case of shock waves entering the domain arising between left and right states with characteristic speed of opposite sign.

One may note that in proposition 2 , the term $u_{l}$ corresponds to the boundary condition at boundary $a$ and to the boundary trace at boundary $b$. Similarly the term $u_{r}$ corresponds to the boundary trace at boundary $a$ and to the boundary condition at boundary $b$.

A graphical representation of the control space for the left boundary $a$ is presented in figure 2, in the case of the Burgers flux, i.e. a quadratic convex flux with minimum at 0 . Cases for which the minimum is not zero can be retrieved by translation. For a general smooth strictly convex flux, a similar representation is obtained with lines replaced by curves in the figure. Figure 2 illustrates the following distinct interactions between the boundary condition, or control, and the boundary trace, or observed value.

- In the domain of weak boundary conditions with negative wave speed arising at the boundary (white zone on the left of vertical axis), the control does not apply. If the upstream value $u(t, a)$ is observed, and a control is applied such that the couple $u(t, a), u_{a}(t)$ is represented by the point $A$, no actuation happens. The trace $u(t, a)$ does not change, it is not impacted by the control chosen. Similarly, no actuation is possible at points $B$ and $C$, and in general in the left white zone. 


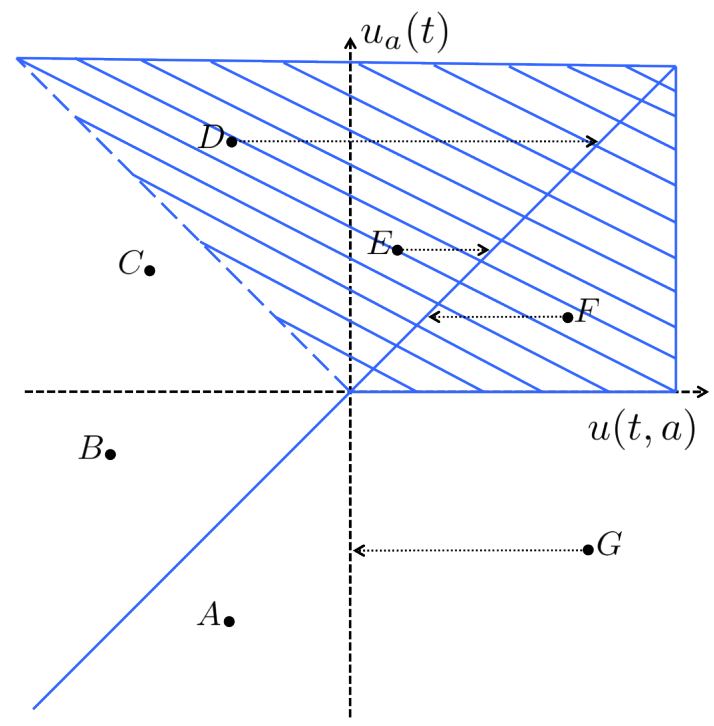

Figure 2: Control space: in the case of a convex quadratic flux with minimum at 0 . Top-right quadrant, first bisector, and upper part of top left quadrant (points $D, E, F)$ : the control applies, i.e. the boundary trace takes the value of the boundary condition, as represented by the horizontal arrow. Left zone except first bisector (points $A, B, C$ ): the control has no effect. Bottom right quadrant (point $G$ ): any control yields a zero boundary trace, represented by a horizontal projection onto the vertical axis.

- In the top right quadrant and upper part of top left quadrant of the control space (striped zone), corresponding to line 2 and 3 of equation (21) respectively, a control action applies; the trace $u(t, a)$ of the solution takes the control value. If a control is applied to a boundary trace value such that the pair is represented by the point $D$, the trace instantaneously takes the value of the control and the resulting configuration is the projection of $D$ onto the first bisector. Similar behavior occurs with the points $E$ and $F$, which belong to the control space. The part of the bottom left quadrant such that the control and the trace of the solution are equal is also part of the strong boundary conditions domain according to definition 6 and its characterization in proposition 2.

- In the bottom right quadrant, any control action yields a vanishing boundary trace, which is illustrated in the case of the application of a control $u_{a}(t)$ such that the couple $\left(u(t, a), u_{a}(t)\right)$ is represented by the point $G$. The trace of the solution $u(t, a)$ takes the value 0 , as illustrated by a horizontal projection onto the axis $x=0$. The control has an action, but not the one intended (i.e. applied), thus the bottom right quadrant is not part of the control space (see definition 6).

Using the characterization of the control space introduced in this section, we show in the following section that the system is stabilizable. 


\subsection{Lyapunov stabilization}

In this section, we prove that there exist boundary conditions in the control space (21)-(22) such that the candidate Lyapunov function (15) is strictly decreasing.

Lemma 3 Let $g: u \mapsto\left(u-u^{*}\right) f(u)-F(u)$, with $f$ a smooth strictly convex function, and $F$ a primitive of $f$. Let $m$ denote the minimum of $f$. The function $g$ is smooth on the real line, and satisfies the following properties:

- $g$ is strictly increasing in $\left(-\infty, \min \left(m, u^{*}\right)\right)$, strictly decreasing in $\left(\min \left(m, u^{*}\right), \max \left(m, u^{*}\right)\right)$, and strictly increasing in $\left(\max \left(m, u^{*}\right),+\infty\right)$.

- For $u>v$ such that $f(u)=f(v)$, we have $g(u)>g(v)$.

Proof 5 The fact that $g$ is smooth results from the smoothness of $f$. The first property is obtained by computing the derivative $g^{\prime}(u)=\left(u-u^{*}\right) f^{\prime}(u)$ of $g$, and noting that $f$ is strictly convex with minimum at $m$. To prove the second property, let us consider $u>v$ such that $f(u)=f(v)$. The difference $g(v)-g(u)$ reads $g(v)-g(u)=F(u)-F(v)+(v-u) f(u)$ that is strictly negative by strict convexity of $f$.

The function $g$ is represented for the case of the Burgers flux function in figure 3 with the arbitrary choice of $g(m)=0$.
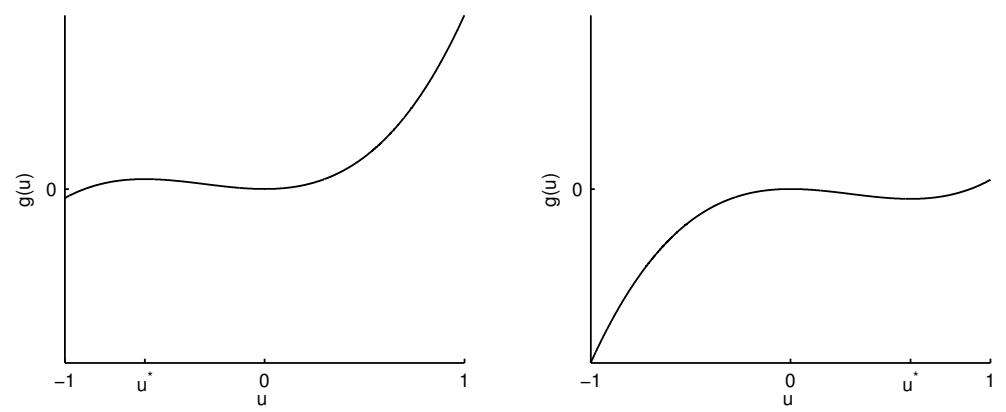

Figure 3: Representation of the variations of $g$ : for a Burgers flux function $f$ in the case $u^{*}<0$ (left) and in the case $u^{*}>0$ (right). The points $u=m$ ( $m=0$ in this case) and $u=u^{*}\left(u^{*}= \pm 0.5\right.$ in this case) are local extrema of $g$.

Theorem 4 Let $V(\cdot)$ denote the candidate Lyapunov function (15) for the PDE (1). There exist boundary conditions $u_{a}(\cdot), u_{b}(\cdot)$ in the control spaces (21) (22), respectively, such that the following holds. If the corresponding solution to the $I B V P$ is in the class $P W S^{+}$then the function $V(\cdot)$ is strictly decreasing, thus the solution is stable in the sense of Lyapunov.

Proof 6 We show that at any given time $t>0$, it is always possible to choose controls $u_{a}(t), u_{b}(t)$ in the control space such that there holds $g(u(t+, a))<$ $g(u(t+, b))$. We consider the case of $u^{*}<m$, and recall that the derivation below correspond to the case of a strictly convex flux. 
- If $u(t, a) \geq m$ and $u(t, b)>m$ then any boundary condition $u_{a}(t) \geq m$ is in the upstream control space. Since $g$ is strictly increasing in $[m ; \infty)$, with $u_{b}(t)=u(t, b)$, it is possible to obtain the strict decrease of the Lyapunov function by choosing $u_{a}(t)$ in $[m, u(t, b)[$.

- If $u(t, a) \geq m$ and $u(t, b) \leq m$ then any boundary conditions $u_{a}(t) \geq m$, $u_{b}(t) \leq m$ are in the control space. Since $g$ is decreasing in $\left[u^{*}, m\right]$ it is possible to choose boundary controls in the control space that guarantees $g\left(u_{a}(t)\right)<g\left(u_{b}(t)\right)$, e.g. $u_{a}(t)=m, u_{b}(t)=u^{*}$.

- If $u(t, a)<m$ and $u(t, a) \neq u^{*}$ and $u(t, b) \leq m$ any boundary condition $u_{b}(t) \leq m$ is in the downstream control space. Since $u^{*}$ is a local maximum of $g$, it is possible to obtain the strict decrease of the Lyapunov function.

- If $u(t, a)=u^{*}$ and $u(t, b) \leq m$ the choice $u(t, b)=u^{*}$ gives a neutral effect of the boundary terms on the Lyapunov function. By assumption we know that the solution $u$ is in $P W S^{+}$, hence in this case either the solution is identically equal to $u^{*}$ or contains shock waves which yields a strictly decreasing Lyapunov function.

- If $u(t, a)<m$ and $u(t, b)>m$,

- If $g(u(t, a))<g(u(t, b))$ the choice $u_{a}(t)=u(t, a)$ and $u_{b}(t)=u(t, b)$ in the control space leads to the strict decrease of the Lyapunov function.

- If $g(u(t, a)) \geq g(u(t, b))$ and $u(t, a) \neq u^{*}$, since $u^{*}$ is a local maximum of $g$ and according to second result of lemma 3 , it is possible to pick $u_{b}(t) \leq m$ such that $f\left(u_{b}\right)>f(u(t, b))$ and $g\left(u_{b}\right)>g(u(t, a))$.

- If $g(u(t, a)) \geq g(u(t, b))$ and $u(t, a)=u^{*}$, the choice $u_{b}(t)=u^{*}$ is in the downstream control space and yields a neutral effect of the boundary terms. Similarly to above, by assumption on the regularity of the solution, we have either the solution identically equal to $u^{*}$ or the Lyapunov function strictly decreasing.

The case $u^{*}>m$ can be treated similarly. The case $u^{*}=m$ can be treated similarly, however involves greater reliance on the internal dynamics. Specifically internal dynamics are the sole contributor to the decrease of the Lyapunov function in the case 2 and 4 above, and the controller is passive (equal to the boundary trace) in the case 5 above.

Remark 4 The set of boundary values $\left(u_{a}(t), u_{b}(t)\right)$ which guarantee stabilization, as described in the proof of Theorem 4 (in the case $u^{*}<m$ ), is defined as follows:

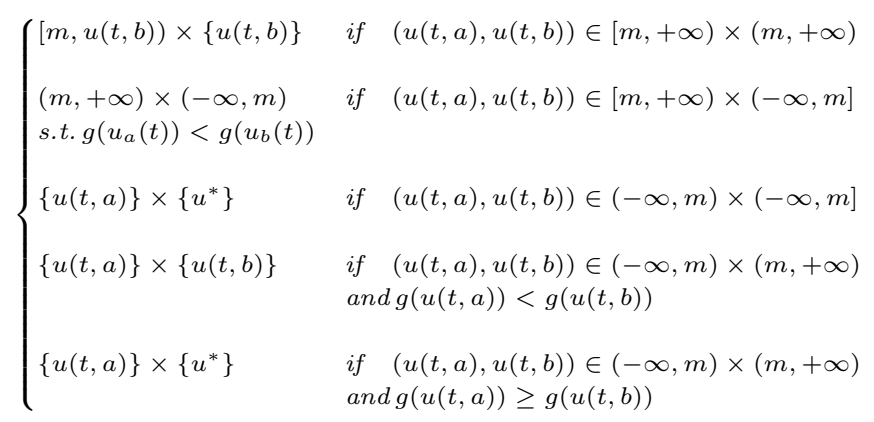


We highlight that the boundary values (23) only provide stability, and not asymptotic stability in general. In the following section we instantiate a greedy controller which maximizes the instantaneous decrease of the Lyapunov function, and also illustrate that the greedy controller may fail to provide asymptotic stability.

In Section 7 we then design an improved controller which guarantees asymptotic stability and we show that the solution resulting from these boundary controls and an initial condition in the class $P W S^{+}$remains in $P W S^{+}$.

\section{Maximizing instantaneous Lyapunov function decrease rate}

In this section, we characterize the values of the control, in the control space, that minimize the Lyapunov function derivative. Since boundary controls introducing a shock wave contribute with a negative term to the internal dynamics, we propose to first characterize the type of wave introduced in specific regions of the control space.

\subsection{Nature of the waves created by boundary control}

The type of wave created at the boundary impacts the value of the derivative of the Lyapunov function. In the scalar case, the type of wave arising is defined by the Lax entropy condition (9). This allows us to partition the control space according to the type of wave introduced by the control. Table 1 summarizes for the upstream boundary the cases in which the boundary control belongs to the control space, with mention of the type of wave introduced.

\begin{tabular}{|l|l|l|}
\hline$u_{a} \geq m$ & $u(t, a)<m$ & $u(t, a) \geq m$ \\
\hline $\begin{array}{l}f\left(u_{a}\right) \\
f(u(t, a)): \\
\text { Shock }\end{array}$ & $u_{a}>u(t, a):$ Shock \\
& $\begin{array}{l}u_{a}=u(t, a): \text { No wave } \\
u_{a}<u(t, a): \text { Rarefac- } \\
\text { tion wave }\end{array}$ \\
\hline$u_{a}<m$ & $\begin{array}{l}u_{a}=u(t, a): \text { No } \\
\text { wave }\end{array}$ & $\begin{array}{l}u_{a} \in \emptyset \\
\text { Rarefaction with van- } \\
\text { ishing boundary trace }\end{array}$ \\
\hline
\end{tabular}

Table 1: Upstream boundary: admissible boundary controls and corresponding wave created at the upstream boundary based on the value of the trace of the solution at this boundary.

From proposition 1, we have that the internal dynamics resulting from the existence of entropic shock waves contributes to the decrease of the Lyapunov function. Here we characterize the benefits of the change in the number of shock 
waves in the solution, resulting from internal interaction, and entrance or exit of a discontinuity at the boundary.

Proposition 3 At a time $t$ at which the number of jump discontinuities $N(t)$ changes:

- If two shock waves interact, the derivative of the Laypunov function decreases.

- If a discontinuity crosses the left boundary, let us note $u^{-}$the value of the boundary trace at time $t^{-}$and $u^{+}$the value of the boundary trace at time $t^{+}$. The jump in the derivative of the Lyapunov function reads

$$
S\left(u^{-}, u^{+}\right) \doteq \frac{d V}{d t}\left(t^{+}\right)-\frac{d V}{d t}\left(t^{-}\right)=\left(f\left(u^{+}\right)-f\left(u^{-}\right)\right) \frac{\tilde{u}^{-}+\tilde{u}^{+}}{2},
$$

in which the term $\left(f\left(u^{+}\right)-f\left(u^{-}\right)\right)$can be checked to be always positive for a convex flux and always negative for a concave flux. Hence the jump in the derivative of the Lyapunov function depends on the sign of $-\left(\tilde{u}^{-}+\tilde{u}^{+}\right) / 2$ $=u^{*}-\left(u^{-}+u^{+}\right) / 2$ :

- If $u^{*}-\left(u^{-}+u^{+}\right) / 2<0$, entering and exiting shocks increase the derivative of the Lyapunov function in the case of a convex flux and decrease the derivative of the Lyapunov function in the case of a concave flux.

- If $u^{*}-\left(u^{-}+u^{+}\right) / 2>0$, entering and exiting shocks decrease the derivative of the Lyapunov function in the case of a convex flux and increase the derivative of the Lyapunov function in the case of a concave flux.

The case of right boundary can be treated similarly changing the sign in (24).

Proof 7 The result is proven in the appendix.

In the following section, we leverage these results to design a stabilizing boundary controller that maximizes the instantaneous decrease rate of the Lyapunov function.

\subsection{Greedy boundary control}

The boundary control that maximizes the decrease rate of the Lyapunov function is the boundary control in the control space, that either introduces a rarefaction wave and minimizes the jump in the Lyapunov function derivative (18), or introduces a shock wave at the boundary and minimizes the jump in the Lyapunov function derivative resulting from the change in the boundary trace and in the number of shock waves (24). The corresponding optimization problem can be formulated as follows.

Proposition 4 Let u denote the solution to the IBVP associated with the scalar conservation law (1). The upstream boundary control $u_{a}^{r}$ and downstream bound- 
ary control $u_{b}^{r}$ that minimize the decrease of the Lyapunov function by introducing rarefaction waves or no waves at the boundary can be obtained by solving

$$
\begin{aligned}
& u_{a}^{r} \doteq \arg \min _{\left\{u \mid(u, u(t, a)) \in \mathcal{C}_{a} \text { and } u \leq u(t, a)\right\}} g(u) \\
& u_{b}^{r} \doteq \arg \max _{\left\{u \mid(u(t, b), u) \in \mathcal{C}_{b} \text { and } u \geq u(t, b)\right\}} g(u) .
\end{aligned}
$$

The upstream boundary control $u_{a}^{s}$ and downstream boundary control $u_{b}^{s}$ that minimize the decrease of the Lyapunov function by introducing discontinuities at the boundary, can be obtained by solving

$$
\begin{aligned}
& u_{a}^{s} \doteq \arg \min _{\left\{u \mid(u, u(t, a)) \in \mathcal{C}_{a} \text { and } u>u(t, a)\right\}} S(u(t, a), u) \\
& u_{b}^{s} \doteq \arg \max _{\left\{u \mid(u(t, b), u) \in \mathcal{C}_{b} \text { and } u<u(t, b)\right\}} S(u(t, b), u) .
\end{aligned}
$$

Proof 8 This results from the characterization of the cases when the boundary control introduces a rarefaction wave or a shock wave, and the previous analysis on the resulting evolution of the Lyapunov function derivative.

Based on the expressions from Proposition 4 and using formula (23), we exhibit a greedy boundary control $\left(u_{a}(t), u_{b}(t)\right)$ maximizing the instantaneous decrease of the Lyapunov function, reading as follows (for the case $u^{*}<m$ ):

$$
\begin{cases}\{m\} \times\{u(t, b)\} & \text { if } \quad(u(t, a), u(t, b)) \in[m,+\infty) \times(m,+\infty) \\ \{m\} \times\left\{u^{*}\right\} & \text { if } \quad(u(t, a), u(t, b)) \in[m,+\infty) \times(-\infty, m] \\ \{u(t, a)\} \times\left\{u^{*}\right\} & \text { if } \quad(u(t, a), u(t, b)) \in(-\infty, m) \times(-\infty, m] \\ & \text { if } \quad(u(t, a), u(t, b)) \in(-\infty, m) \times(m,+\infty) \\ \{u(t, a)\} \times\{u(t, b)\} & \text { and } g(u(t, b))>g\left(u^{*}\right) \\ & \text { if } \quad(u(t, a), u(t, b)) \in(-\infty, m) \times(m,+\infty) \\ \{u(t, a)\} \times\left\{u^{*}\right\} & \text { and } g(u(t, b)) \leq g\left(u^{*}\right)\end{cases}
$$

and the action of the controller are illustrated in Figure 4. While the greedy controller (25) maximizes the instantaneous decrease of the Lyapunov function, we illustrate in the following example that asymptotic stability may not be obtained. We also illustrate the naive brute force control $\left(u_{a}(t)=u^{*}, u_{b}(t)=\right.$ $u^{*}$ ) may create oscillations at the boundary, in the form of infinite variation in time of the solution trace.

Example 1 Without loss of generality, we choose $u^{*}<m$ and define $\hat{u}$ by $u^{*}<m<\hat{u}$ and $f(\hat{u})=f\left(u^{*}\right)$ Given $0<\Delta<(a+b) / 2$, such that $\frac{b-a}{4 \Delta} \in \mathbb{N}$, and $0<k<\hat{u}$ we consider the following initial datum on $(a, b)$ :

$$
u_{0}(x)=\left\{\begin{array}{ll}
m & \text { if } x \in\left(a, \frac{a+b}{2}\right) \\
\hat{u}-k & \text { if } x \in\left(\frac{a+b}{2}+(2 p) \Delta, \frac{a+b}{2}+(2 p+1) \Delta\right) \\
& p \in\left\{0, \cdots, \frac{b-a}{4 \Delta}-1\right\} \\
\bar{u}+k & \text { if } x \in\left(\frac{a+b}{2}+(2 p+1) \Delta, \frac{a+b}{2}+(2 p+2) \Delta\right) \\
& p \in\left\{0, \cdots, \frac{b-a}{4 \Delta}-1\right\}
\end{array} .\right.
$$




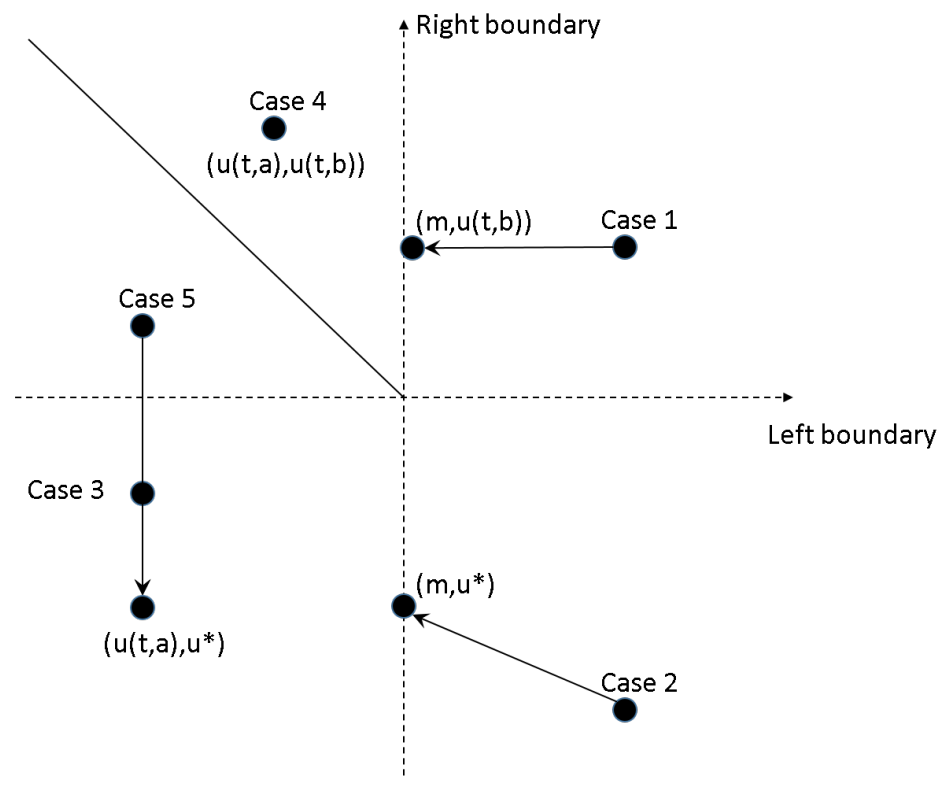

Figure 4: Greedy stabilizing controller: in the case of a quadratic convex flux centered at 0 , for $u^{*}<m$. The cases correspond to the rows of the controller expression in equation (25).

This case corresponds to the first row of equation (25), hence the applied boundary controls is $\left(u_{a}(t)=m, u_{b}(t)=u(t, b)\right)$. Since the characteristic speed of $m$ is zero, the right boundary value converges towards $m$ only in infinite time, hence the system remains in the configuration characterized by the first row of equation (25), and converges to the steady state $m$ over the interval $(a, b)$, not reaching the target $u^{*}<m$. Hence we have stability but not asymptotic stability.

For the same example, one may note that the brute force control $\left(u_{a}(t)=\right.$ $\left.u^{*}, u_{b}(t)=u^{*}\right)$ has no action on the system when $u(t, b)=\bar{u}+k$ since the control values are outside of the control space. While when $u(t, b)=\bar{u}-k$, the brute force control induces slow backward moving shock waves $\left(\bar{u}-k, u^{*}\right)$ from the right boundary which interact with fast forward moving shock waves $(\bar{u}+k, \bar{u}-k)$ coming from the initial datum, and create slow forward moving shock waves $\left(\bar{u}+k, u^{*}\right)$, hence we observe large oscillations at the right boundary (irrespective of the size of the oscillations in the initial datum). More precisely, the trace at the boundary $x=a$ oscillates between the value $u^{*}$ and values in the interval $[\hat{u}-k, \hat{u}+k]$, generating a total variation in time which satisfies: $T V_{t}\left(u_{a}\right) \geq \frac{b-a}{4 \Delta}\left(\hat{u}-k-u^{*}\right)$. Since $\Delta$ is arbitrary this oscillation can not be bounded from above, even for $k$ arbitrarily small. In other words, we can produce arbitrarily big oscillations with initial data $u_{0}$ of uniformly bounded variation in space. Eventually all the waves generating by oscillating initial datum exit the domain and the naive control produces backward moving shock waves $\left(m, u^{*}\right)$ which yield convergence.

In the following section we present an improved controller providing Lyapunov asymptotic stability. 


\section{$7 \quad$ Lyapunov asymptotic stability}

In this section we design an improved controller which guarantees asymptotic stability and show that the associated solution to the corresponding IBVP remains in the class $\mathrm{PWS}^{+}$for initial data $u_{0}$ in the same class.

\subsection{Controller design}

The asymptotic convergence issue highlighted in the Example 1 stems from the fact that if the system reaches a configuration corresponding to the first row of equation (25), given the prescribed control values, it may remain in that configuration, which grants stability but prevents asymptotic stability for $u^{*}<m$. Without loss of generality, we focus on the case $u^{*}<m$. We define $\hat{u}>m$ by $f(\hat{u})=f\left(u^{*}\right), \check{u}>m$ by $g(\check{u})=g(u *)$ and $\bar{u}=\frac{\hat{u}+\check{u}}{2}$. We propose the following values for the controller $\left(u_{a}(t), u_{b}(t)\right)$ :

$$
u_{a}(t)=\min \{u(t, a), m\}, \quad u_{b}(t)=\psi(u(t, b)),
$$

where $\psi(\alpha)=\chi_{]-\infty, \bar{u}]}(\alpha) u^{*}+\left(1-\chi_{]-\infty, \bar{u}]}(\alpha)\right) \alpha$ and $\chi_{I}$ is the indicator function of the interval $I$. It is convenient to describe the control according to different cases as for the greedy control, thus we get:

$$
\left\{\begin{array}{lll}
\{m\} \times\{u(t, b)\} & \text { if } & (u(t, a), u(t, b)) \in[m,+\infty) \times(\bar{u},+\infty) \\
\{m\} \times\left\{u^{*}\right\} & \text { if } & (u(t, a), u(t, b)) \in[m,+\infty) \times(-\infty, \bar{u}] \\
\{u(t, a)\} \times\left\{u^{*}\right\} & \text { if } & (u(t, a), u(t, b)) \in(-\infty, m) \times(-\infty, \bar{u}] \\
\{u(t, a)\} \times\{u(t, b)\} & \text { if } & (u(t, a), u(t, b)) \in(-\infty, m) \times(\bar{u},+\infty)
\end{array}\right.
$$

\subsection{Existence of solution for asymptotically stabilizing con- troller}

To apply Theorem 4 , we need to define boundary controls $u_{a}$, $u_{b}$, which will guarantee the solution to the corresponding IBVP to remain in the class $\mathrm{PWS}^{+}$ for initial data $u_{0}$ in the same class. We prove such result for the boundary control providing asymptotic stability of the Lyapunov function (15). For simplicity we assume $u^{*}<m$ being the other case similar.

Theorem 5 Consider an initial datum $u_{0}$ in $P W S^{+}$and the boundary controls given by formula (27). Then the corresponding IBVP admits a unique solution which is in the class $P W S^{+}$for all times.

Proof 9 We will show that for $u_{0}$ in $P W S^{+}$, the boundary controls are well defined and piecewise smooth in time. Moreover the solution remains in the class $P W S^{+}$.

First we enumerate the following cases:

- Case 1: $u(t, a) \geq m, u(t, b)>\bar{u}$.

- Case 2: $u(t, a) \geq m, u(t, b) \leq \bar{u}$.

- Case 3: $u(t, a)<m, u(t, b) \leq \bar{u}$. 
- Case $4: u(t, a)<m, u(t, b)>\bar{u}$.

In Case 1 the boundary controls generate a rarefaction wave from the left boundary, no wave on the right boundary and the traces verify $u(t+, a)=m$ and $u(t+, b)=u(t, b)>\bar{u}>m$ (where $u(t+, \cdot)$ indicates the limit at time $t$ from the above).

In Case 2 the boundary controls generate a rarefaction wave from the left boundary, a rarefaction wave or a shock from the right boundary and the traces verify $u(t+, a)=m$ and $u(t+, b)=u^{*}$.

In Case 3 the boundary controls generate no wave from the left boundary, a rarefaction wave or a shock on the right boundary and the traces verify $u(t+, a)=$ $u(t, a)<m$ and $u(t+, b)=u^{*}$.

In Case 4 the boundary controls generate no wave from the left and right boundary and the traces verify $u(t+, a)=u(t, a)<m$ and $u(t+, b)=u(t, b)>\bar{u}>m$. From this analysis we verify that the control is well defined for constant traces and the corresponding solution remains in the class $P W S^{+}$. We are left to verify that the solution is well defined and remains in the same class for waves interacting with the boundary and we proceed again by cases.

In case 1: if a wave interacts with the left boundary, then no wave is produced, while for the right boundary a shock may arise and we transition to case 2. In case 2: if a wave interacts with the left or right boundary, then no wave is produced and we transition to case 1 or case 3. In case 3: if a wave interacts with the left or right boundary, then no wave is produced and we remain in case 3 or transition to case 4. In case 4: if a wave interacts with the left boundary, then no wave is produced, while for the right boundary a shock may arise and we transition to case 3. This concludes the analysis of interacting waves and the proof.

In order to extend our results to initial data $u_{0}$ in BV (and not necessarily in $\mathrm{PWS}^{+}$) we provide estimates on the total variation in time of the controls $T V_{t}\left(u_{a}\right), T V_{t}\left(u_{b}\right)$, and in space of the generated solution $T V_{x}(u(t, \cdot))$.

Theorem 6 Consider an initial datum $u_{0}$ in $P W S^{+}$, the boundary controls given by formula (25) and let us indicate by $u(t, x)$ the corresponding solution. Then, defining $C=2\left(\sup _{x}\left|u_{0}(x)-m\right|+\left|m-u^{*}\right|\right)$, we have the following estimates:

$$
\begin{gathered}
T V_{x}(u(t, \cdot)) \leq T V_{x}\left(u_{0}\right)+C+\left|\bar{u}-u^{*}\right| \\
T V_{t}\left(u_{a}\right) \leq T V_{x}\left(u_{0}\right)+C \\
T V_{t}\left(u_{b}\right) \leq T V_{x}\left(u_{0}\right)+C+\left|\bar{u}-u^{*}\right| \cdot \frac{T V_{x}\left(u_{0}\right)+C}{|\bar{u}-\hat{u}|} .
\end{gathered}
$$

Proof 10 Let us start showing that:

$$
T V_{x}(u(0+, \cdot)) \leq T V_{x}\left(u_{0}\right)+C .
$$

We consider cases 1, 2, 3 and 4 as in the proof of Theorem 5. In case 1 a wave is generated from the left boundary of strength $|u(t, a)-m|$; in case 2 a wave is generated from the left boundary of strength $|u(t, a)-m|$ and a wave from the right boundary of strength $\left|u(t, b)-u^{*}\right| \leq|u(t, b)-m|+\left|m-u^{*}\right|$; in case 3 a wave is generated from the right boundary of strength $\left|u(t, b)-u^{*}\right|$; in case 4 no wave is generated from the boundaries. We thus obtained the desired estimate. 
We now pass to the other estimates dealing with wave interactions by cases as in the proof of Theorem 5. We use the symbol $\Delta$ to indicate the change in total variation due to the interaction. For instance if $\bar{t}>0$ is the interaction time of a wave then $\Delta T V_{t}\left(u_{a}\right)=T V_{t}\left(u_{a} ;[0, \bar{t}]\right)-T V\left(u_{a} ;[0, \bar{t}[)\right.$.

In case 1 if a wave $(u(t, a), \tilde{u})$ interacts with the left boundary, then, for the wave to have negative speed, we must have $\tilde{u}<m=u(t, a)$. Therefore no wave is generated from the boundaries and we transition to case 4. Moreover, we get:

$$
\begin{aligned}
& \Delta T V_{x}(u(t, \cdot))=-|u(t, a)-\tilde{u}|<0, \\
& \Delta T V_{a}=|u(t, a)-\tilde{u}|, \quad \Delta T V_{b}=0 .
\end{aligned}
$$

In case 1 if a wave $(\tilde{u}, u(t, b))$ interacts at time $\bar{t}$ with the right boundary, then we distinguish two cases. If $\tilde{u}>\bar{u}$ no wave is generated, we remain in case 1 and we get:

$$
\begin{aligned}
& \Delta T V_{x}(u(t, \cdot))=-|u(t, b)-\tilde{u}|<0, \\
& \Delta T V_{b}=|u(t, b)-\tilde{u}|, \quad \Delta T V_{a}=0 .
\end{aligned}
$$

Otherwise, if $m \leq \tilde{u} \leq \bar{u}$, then the shock $\left(\tilde{u}, u^{*}\right)$ is generated from the right boundary, we transition to case 2 and we get:

$$
\begin{gathered}
\Delta T V_{x}(u(t, \cdot))=\left|u^{*}-\tilde{u}\right|-|u(t, b)-\tilde{u}| \leq\left|\bar{u}-u^{*}\right|, \\
\Delta T V_{t}\left(u_{a}\right)=0, \\
\begin{aligned}
\Delta T V_{t}\left(u_{b}\right)= & \left|u(t, b)-u^{*}\right| \leq|u(t, b)-\tilde{u}|+\left|\tilde{u}-u^{*}\right| \\
\leq & T V_{x}(u(\bar{t}-, \cdot))+\left|\bar{u}-u^{*}\right| .
\end{aligned}
\end{gathered}
$$

In case 2 if a wave $(u(t, a), \tilde{u})$ interacts with the left boundary, then no wave is generated from the boundaries and we transition to case 3. Moreover we have, as for the case 1:

$$
\begin{aligned}
& \Delta T V_{x}(u(t, \cdot))=-|u(t, a)-\tilde{u}|<0, \\
& \Delta T V_{a}=|u(t, a)-\tilde{u}|, \quad \Delta T V_{b}=0 .
\end{aligned}
$$

In case 2 if a wave $(\tilde{u}, u(t, b))$ interacts at time $\bar{t}$ with the right boundary, then, for the wave to have positive speed, we must have $\tilde{u}>\hat{u}>\bar{u}$. Thus no wave is generated and we transition to case 1. Moreover, we get:

$$
\begin{aligned}
& \Delta T V_{x}(u(t, \cdot))=-|u(t, b)-\tilde{u}|<0, \\
& \Delta T V_{b}=|u(t, b)-\tilde{u}|, \quad \Delta T V_{a}=0 .
\end{aligned}
$$

For case 3 we have the same analysis as case 2. More precisely, if a wave interacts with the left boundary no wave is generated and we remain in case 3, while if a wave interacts with the right boundary then no wave is generated and we transition to case 4. Moreover we get the same estimates for the total variations.

In case 4 if a wave $(u(t, a), \tilde{u})$ interacts with the left boundary, then, for the wave to have negative speed, we must have $\tilde{u}<m$. Therefore no wave is generated from the boundaries and we remain in case 4. Moreover, we get:

$$
\Delta T V_{x}(u(t, \cdot))=-|u(t, a)-\tilde{u}|<0
$$




$$
\Delta T V_{a}=|u(t, a)-\tilde{u}|, \quad \Delta T V_{b}=0 .
$$

In case 1 if a wave $(\tilde{u}, u(t, b))$ interacts at time $\bar{t}$ with the right boundary, then we distinguish two cases. If $\tilde{u}>\bar{u}$ no wave is generated, we remain in case 4 and we get:

$$
\begin{aligned}
& \Delta T V_{x}(u(t, \cdot))=-|u(t, b)-\tilde{u}|<0, \\
& \Delta T V_{b}=|u(t, b)-\tilde{u}|, \quad \Delta T V_{a}=0 .
\end{aligned}
$$

Otherwise, if $\tilde{u} \leq \bar{u}$, then the shock $\left(\tilde{u}, u^{*}\right)$ is generated from the right boundary, we transition to case 3 and we get:

$$
\begin{gathered}
\Delta T V_{x}(u(t, \cdot))=\left|u^{*}-\tilde{u}\right|-|u(t, b)-\tilde{u}| \leq\left|\bar{u}-u^{*}\right|, \\
\Delta T V_{t}\left(u_{a}\right)=0, \\
\begin{aligned}
\Delta T V_{t}\left(u_{b}\right)= & \left|u(t, b)-u^{*}\right| \leq|u(t, b)-\tilde{u}|+\left|\tilde{u}-u^{*}\right| \\
\leq & T V_{x}(u(\bar{t}-, \cdot))+\left|\bar{u}-u^{*}\right| .
\end{aligned}
\end{gathered}
$$

The results of the estimates are summarized in Figure 5. It is easy to notice

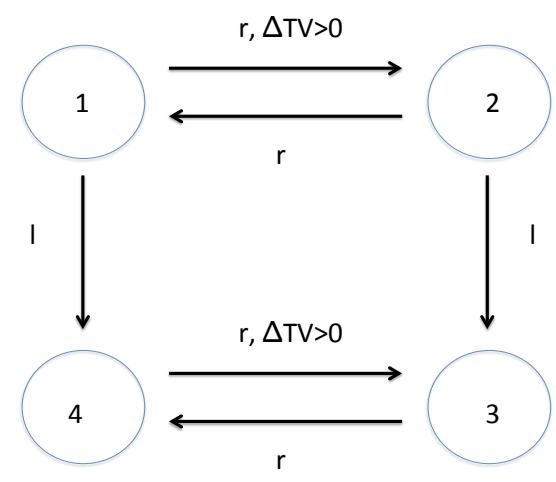

Figure 5: Graphical representation of transitions between cases 1 to 4 . The arrow labels indicate: the boundary reached by the interacting wave, $l$ for left and $r$ for right, and $\Delta T V>0$ if the total variations may increase. Transitions from a case to itself are not represented for simplicity.

that transitions from cases 1,2 to cases 3,4 are irreversible. Therefore, multiple increases in total variation can occur only for transitions 1-2-1 (that is from case 1 to case 2 and then back to case 1) and for transitions 4-3-4. We prove estimates for the transitions 1-2-1, being the case 4-3-4 entirely similar. We denote by $\left(\tilde{u}_{1}, u_{b}^{1}\right)$ the interacting wave for transition 1-2 and by $\left(\tilde{u}_{2}, u_{b}^{2}=u^{*}\right)$ the interacting wave for the transition 2-1. Notice that $m \leq \tilde{u} \leq \bar{u}<u_{b}^{1}$ and $\bar{u}<\hat{u}<\tilde{u}_{2}$. Again we use the symbol $\Delta$ to indicate the change in total variations before and after the transition 1-2-1. Using the above estimates, we get:

$$
\begin{aligned}
\Delta T V_{x}(u(t, \cdot)) & =\left|u^{*}-\bar{u}\right|-\left|\tilde{u}_{2}-u_{b}^{2}\right| \leq\left|u^{*}-\bar{u}\right|-|\hat{u}-\bar{u}| \\
& \leq-|\hat{u}-\bar{u}|<0 .
\end{aligned}
$$


In other words, each transition 1-2-1 (and similarly 4-3-4) gives rise to a decrease of $T V_{x}(u(t, \cdot))$ which is bounded away from zero. Therefore, we deduce that $T V_{x}(u(t, \cdot))$ is bounded and transitions 1-2-1 can occur only a finite number of times (namely $\left.\frac{T V(u(0+, \cdot))}{|\hat{u}-\bar{u}|}\right)$. Combining this result with the estimates of cases 1, 2, 3 and 4 and (31), we get the estimates (28), (29) and (30).

We are now ready to state last theorem of this section:

Theorem 7 Consider an initial datum $u_{0}$ in $B V$ and the boundary controls given by formula (25), then there exists a unique entropic solution to the corresponding IBVP problem. Moreover, estimates (28), (29) and (30) hold true. Finally we have $\lim _{t \rightarrow+\infty} V(t)=0$, i.e. $\lim _{t \rightarrow+\infty}\left\|u(t, \cdot)-u^{*}\right\|_{L^{2}}=0$.

Proof 11 Consider an approximating sequence $u_{0}^{n}$ of $u_{0}$ such that $u_{0}^{n} \in P W S^{+}$ and $T V\left(u_{0}^{n}\right) \leq T V\left(u_{0}\right)$. Applying Theorem 5, we get the existence of entropic solutions $u^{n}(t, x)$ to the corresponding IBVP problems. Moreover, estimates (28), (29) and (30) hold true for $u^{n}$. By Helly Theorem, there exists a subsequence of $u^{n}$ such that the solution $u^{n}$ and the corresponding boundary controls $u_{a}^{n}(t)$ and $u_{b}^{n}(t)$ converge strongly in $L^{1}$ to some limit $w, w_{a}$ and $w_{b}$. Moreover, estimates (28), (29) and (30) hold true. Therefore, for almost every $t$ we get that $w_{a}(t)=u_{a}(w(t, a+), w(t, b-))$ and $w_{b}(t)=u_{b}(w(t, a), w(t, b))$ (according to formula (25)). We conclude that $w$ is a solution to the IBVP with initial condition $u_{0}$ and boundary controls given by formula (25). Uniqueness follows from standard theory for IBVP.

Because of estimates (29) and (30), there exist the asymptotic limits of boundary controls $w_{a}^{\infty}=\lim _{t \rightarrow+\infty} w_{a}(t)$ and $w_{a}^{\infty}=\lim _{t \rightarrow+\infty} w_{a}(t)$. If $w_{a}^{\infty}>m$, then for $t$ sufficiently big a rarefaction would be generated from left boundary $\left(m, w_{a}^{\infty}\right)$ contradicting the definition of $w_{a}^{\infty}$. Similarly, we must have $w_{b}^{\infty}=u^{*}$ or $w_{b}^{\infty}>\bar{u}$. In all cases the solution $w$ to the IBVP, for $t$ sufficiently large, will attain the boundary values, so solve the conservation law on the whole real line. Now, if $w_{a}^{\infty} \geq w_{b}^{\infty}$ then $w_{b}^{\infty}=u^{*}$ and we can apply Theorem 2 of [61], thus the solution decays to the traveling shock $\left(w_{a}^{\infty}, w_{b}^{\infty}\right)$. However, this traveling shock has negative speed unless $w_{a}^{\infty}=w_{b}^{\infty}=u^{*}$. Thus we conclude that $V(t)$ tends to 0 .

Otherwise, if $w_{a}^{\infty}<w_{b}^{\infty}$ then we can apply Theorem 4 of [61] and deduce that $w$ decays to an $N$-wave formed by two shocks enclosing the rarefaction $\left(w_{a}^{\infty}, w_{b}^{\infty}\right)$. If the first shock has positive speed, then the solution $w$ tends uniformly to $w_{a}^{\infty}$ and we conclude as before. Similarly, if the second shock has negative speed, then the solution $w$ tends uniformly to $w_{b}^{\infty}$ and conclude as before. Finally, if the first shock has negative speed and the second positive speed then they asymptotically exit the domain $[a, b]$ and the solution $w$ decays to the rarefaction $\left(w_{a}^{\infty}, w_{b}^{\infty}\right)$. Now, if $w_{b}^{\infty}>\bar{u}$ then, for $t$ sufficiently big, the characteristics with positive speed of the rarefaction wave, i.e. corresponding to $u \geq m$, will reach the right boundary, contradicting $w_{b}^{\infty}>\bar{u}$. Therefore $w_{b}^{\infty}=u^{*}$. The rarefaction $\left(w_{a}^{\infty}, w_{b}^{\infty}\right)$ is then encompassed of characteristics with negative speed, thus exit the domain, unless $w_{a}^{\infty}=w_{b}^{\infty}=u^{*}$. Thus we conclude that $V(t)$ tends to 0 also in this case.

\subsection{Non-local controls}

As we noticed the greedy control may not stabilize the system to $u^{*}$, while the brute force control $u_{a} \equiv u_{b} \equiv u^{*}$ may overshoot (and not be in the control 
space) and produce oscillations. Finally, control (27) stabilizes the system, but the stabilization time can be far from optimal (and the same is for the brute force ones). Therefore, in this Section, we show a nonlocal control $u_{a, b}^{n l}$ which fast stabilizes the system to $u^{*}$. We use the term nonlocal to indicate that this control will depend not only on the values of the traces $u(t, a)$ and $u(t, b)$.

We focus again, for simplicity, on the case $u^{*}<m$. Let $A=\sup _{x \in[a, b]} u_{0}(x)$ and $\hat{A}<m$ be such that $f(\hat{A})=f(A)$. For every $U<\hat{A}$ we define:

$$
T_{1}(U)=\frac{(b-a)(A-U)}{f(U)-f(A)}, \quad T_{0}(U)=T_{1}-\frac{(b-a)}{\left|f^{\prime}(U)\right|}
$$

and set $u_{a}^{n l}$ as in (27), while:

$$
u_{b}^{n l}(t)=\left\{\begin{array}{ll}
U & 0 \leq t \leq T_{0} \\
u^{*} & T_{0}<t<+\infty
\end{array} .\right.
$$

The meaning of this control is as follows. First we send a large shock $\left(u_{0}(a), U\right)$ with negative speed to move the system in the zone $u<m$ and then apply the stabilizing control. Notice that $T_{1}$ is computed as the maximal time taken by the big shock to cross the interval $[a, b]$, while $T_{0}$ is the time at which the characteristic corresponding to $u^{*}$ should start from $b$ to reach $a$ at time $T_{1}$. These choices will guarantee the desired effect. Notice also that $T_{0}$ is a safe choice, but smaller values may give a better performance. In the following section we present numerical results of the implementation of the boundary control proposed.

\section{Numerical examples}

In this section, we present numerical results obtained for a benchmark scenario. The numerical scheme used is the standard Godunov scheme [42] with 200 cells in space and a time discretization satisfying the tight Courant-Friedrich-Levy (CFL) condition [56]. We consider the flux function $u \mapsto u^{2} / 2$, the equilibrium state $u^{*}=-1$, and the space domain $[0,1]$ with the oscillating initial condition:

$$
u_{0}(x)=1+0.5 \sin (20 x) \text {. }
$$

In figure 6 we present the evolution of the system under four different controllers: the greedy boundary control (defined in proposition 4), the brute force boundary control $u_{a}=u_{b}=u^{*}$, the stabilizing control (formula (25)) and nonlocal control (formula 33) with $U=-2$ and $T_{0}$ as defined in (32). The greedy control allows oscillations to exit from the right boundary but the solution does not converge to the steady state $u^{*}=-1$. On the other side the brute force control converges to the steady state but generates oscillations on the right boundary as can be seen in Figure 7, top. The stabilizing control also converges but it is less oscillating with respect to the brute force control. The non local control guarantees convergence and avoids oscillations. The evolution of the solution under the action of the stabilizing control and the brute force control are very similar. The decrease of the corresponding Lyapunov functions is represented in figure 7 , bottom. One can note how the nonlocal control decreases much faster than the other methods. 

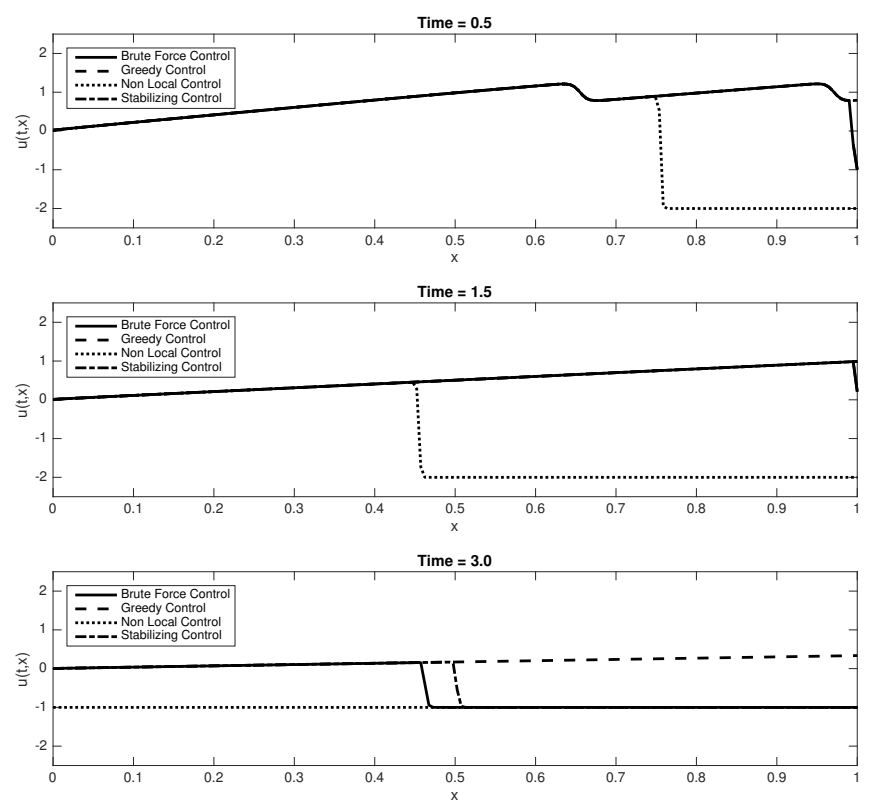

Figure 6: Numerical solution of Burgers equation: Evolution of the solution for the various controllers with oscillating initial data.
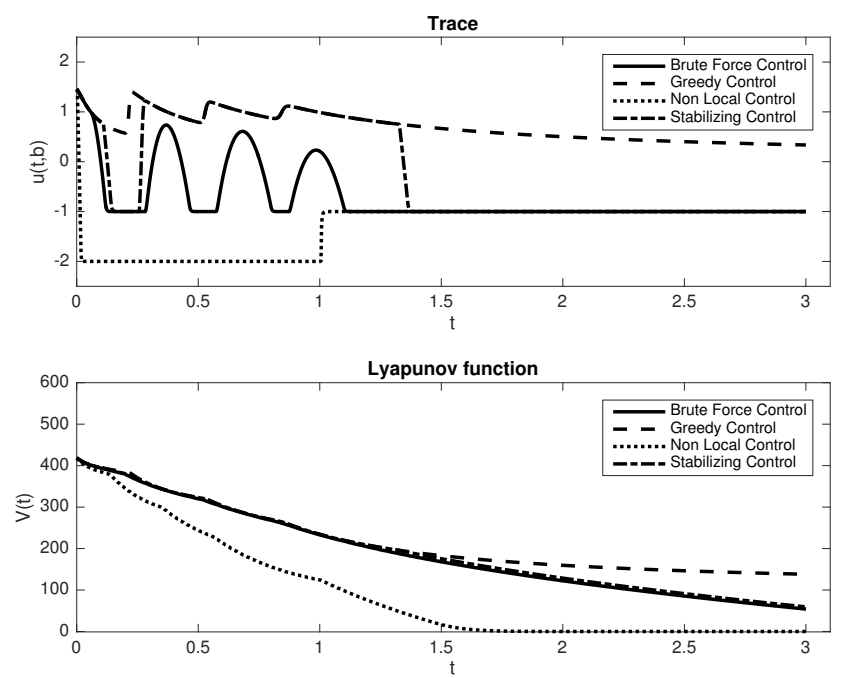

Figure 7: Downstream trace and Lyapunov functions: the Lyapunov functions for different controls are represented in the bottom subfigure. The downstream boundary are represented in the top subfigure respectively. 
To study the dependence of the nonlocal control stabilization performance on the parameters $U$ and $T_{0}$ we run several simulations with different values of these parameters, see figure 8 for $U \in[-2.1,-1.5]$ and $T_{0} \in[0.5,2]$. The convergence time is defined as the first time such that $V(t) \leq 0.1$. We notice that longest convergence time corresponds to $U=-1.5$ and $T_{0}=0.5$ while the fastest corresponds to $U=-2.1$ and $T_{0}=1$. Moreover, for each fixed $U$ there exists an optimal switching time $T_{0}$ that minimizes the convergence time.

To further illustrate the oscillations of the boundary trace generated by the

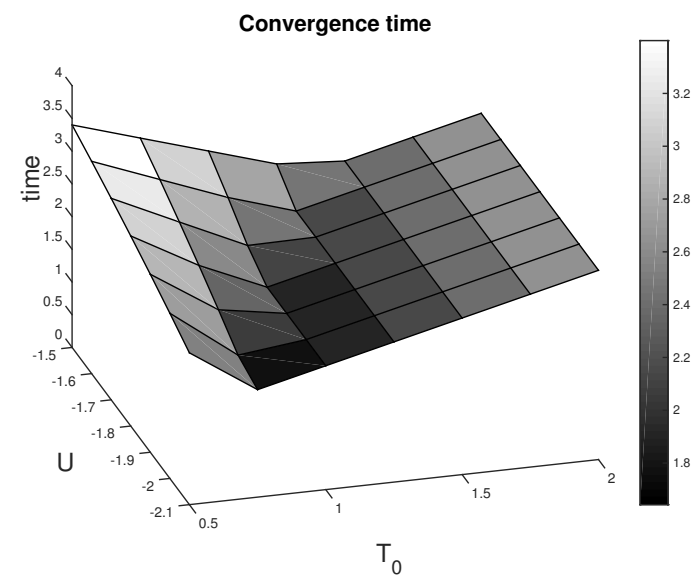

Figure 8: Convergence time of the Lyapunov function: dependence of the convergence time of the Lyapunov function from $U$ and $T_{0}$. The convergence time is defined as the first time for which $V(t) \leq 0.1$.

brute force control we simulated the case in which the initial datum is strongly oscillating:

$$
u_{0}(x)=1+0.3 \sin (50 x) .
$$

In figure 9 the trace of the brute force control shows, at initial times, one big oscillations and then the oscillations continues until $t=1$. For the stabilizing control the oscillations are smaller but they extend for a longer period up to time $t=1.5$.

\section{Conclusion}

In this article, we introduced a new technique for Lyapunov boundary stabilization results of weak entropy solutions to scalar conservation laws.

We proved that under suitable regularity of the initial and boundary data, the solution to the initial-boundary value problem could be considered to be piecewise regular with a finite number of discontinuities. This allows the use of functional analysis tools available for smooth functions.

We then computed the derivative of the Lyapunov function, and showed that the boundary control maximizing its decrease, called greedy control, may fail to stabilize. Therefore, we designed a new stabilizing controller that achieve the desired result. Moreover, we provided estimates of the total variation in space of the corresponding solution and in time of controls, allowing the treatment of 


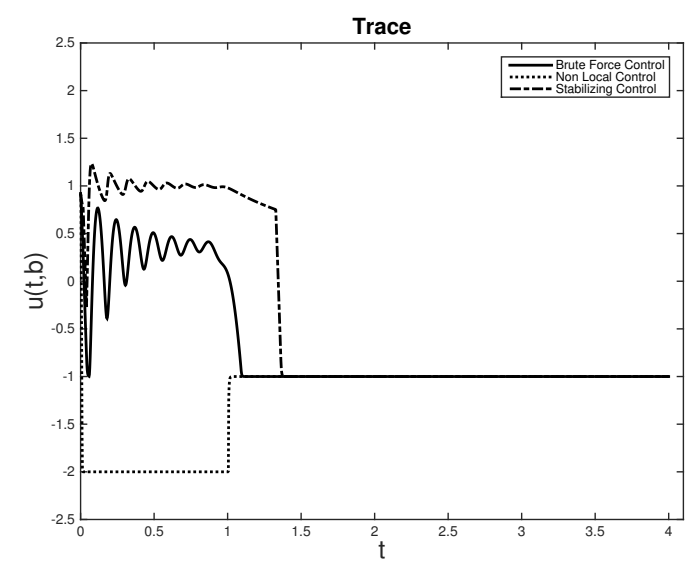

Figure 9: Downstream trace: value of the solution at the right boundary for a strong oscillating initial data.

BV initial data and the proof of $L^{2}$ convergence.

We also introduced nonlocal controls (depending on the whole initial datum) which improve the convergence time.

Numerical results illustrate the various control techniques, including oscillation phenomena introduced by brute force control (setting the boundary value always equal to the desired equilibrium).

Extension to this work include the treatment of non-uniform stationary conditions, and the case of networks, for more applicable results to reliable water distribution and congestion control on the road network.

\section{Acknowledgments}

The authors would like to thank Professor Miroslav Krstic for suggesting to work on this problem during his visit at UC Berkeley and for fruitful conversation leading to the genesis of this work. At this time the second author was a visiting scholar at UC Berkeley with financial support from CEMAGREF and from the France-Berkeley fund project. The authors would also like to thank their colleague Professor Daniel Work from UIUC for numerous fruitful discussions on a related topic.

\section{Appendix}

Proof of proposition 3.

Proof 12 We show each part of proposition 3 separately.

- We show that in the case of $|N(t+)|=|N(t-)|-1$ due to the interaction of two shocks inside the domain, there is a decrease in the slope of the derivative of the Lyapunov function if and only if the Rankine-Hugoniot speed of the left shock is greater than the Rankine-Hugoniot speed of the right shock, which is a necessary condition for the interaction. We compute the difference in the right and left derivative, at time $t$, of the Lyapunov 
function, by taking the difference of equation (18) at time $t^{+}$and $t^{-}$. It is clear that the boundary terms cancel out, and that in the sum terms, only the terms corresponding to the interacting shocks and the created shock do not cancel out. We note $\left(u_{l}^{+}, u_{r}^{+}\right)$the left and right values at the created shock at time $t^{+}$. We use similar notations for the interacting shocks at time $t^{-}$. The left and right states of the left shock at time $t^{-}$are denoted $\left(u_{1 l}^{-}, u_{1 r}^{-}\right)$, and the left and right states of the right shock at time $t^{-}$are denoted $\left(u_{2 l}^{-}, u_{2 r}^{-}\right)$. We note that by continuity outside of the jump locations, we have $u_{1 r}^{-}=u_{2 l}^{-}$, and $u_{1 l}^{-}=u_{l}^{+}$and $u_{2 r}^{-}=u_{r}^{+}$. After some long but straightforward algebra, this allows us to simplify the difference to:

$$
\begin{aligned}
\frac{d V}{d t}\left(t^{+}\right)-\frac{d V}{d t}\left(t^{-}\right)=\frac{1}{2}\left[\left(f\left(u_{1 r}^{-}\right)-\right.\right. & \left.f\left(u_{1 l}^{-}\right)\right)\left(u_{2 l}^{-}-u_{2 r}^{-}\right) \\
& \left.+\left(f\left(u_{2 r}^{-}\right)-f\left(u_{2 l}^{-}\right)\right)\left(u_{1 r}^{-}-u_{1 l}^{-}\right)\right]
\end{aligned}
$$

and the right side is negative if and only if the two shocks intersect, which is satisfied by assumption.

- In the case of a shock entering or exiting from the boundary, we show that the sign of the difference in the right and left derivative, at time $t$, of the Lyapunov function, depends on the sign of $u^{*}-\left(u^{-}+u^{+}\right) / 2$. We compute the difference between the Lyapunov function derivative at time $t^{+}$and at time $t^{-}$. It is clear that only the boundary term at time $t^{+}$and $t^{-}$, and the term corresponding to the entered or exited shock do not cancel out. If we note $u^{-}$the boundary trace at $t^{-}$and $u^{+}$the boundary trace at $t^{+}$, we obtain after simplification:

$$
\frac{d V}{d t}\left(t^{+}\right)-\frac{d V}{d t}\left(t^{-}\right)=\left(f\left(u^{+}\right)-f\left(u^{-}\right)\right) \frac{\tilde{u}^{-}+\tilde{u}^{+}}{2},
$$

for both entering and exiting shocks. We treat the case of a shock entering the domain from upstream boundary for a convex flux. We have $u^{+}>u^{-}$ from the entropy condition, hence from the Rankine-Hugoniot relation we obtain $f\left(u^{+}\right)>f\left(u^{-}\right)$since the shock speed is positive. So the jump in the Lyapunov derivative is negative if and only if $\tilde{u}^{-}+\tilde{u}^{+}<0$, which is equivalent to $\left(u^{-}+u^{+}\right) / 2<u^{*}$.

\section{References}

[1] D. AmADORI. Initial-boundary value problems for nonlinear systems of conservation laws. NoDEA: Nonlinear Differential Equations and Applications, 4(1):1-42, 1997.

[2] D. Amadori and R. Colombo. Continuous dependence for $2 \times 2$ conservation laws with boundary. Journal of Differential Equations, 138(2):229-266, 1997.

[3] D. Amadori and R. Colombo. Viscosity solutions and standard Riemann semigroup for conservation laws with boundary. Rendiconti del Seminario Matematico della Universita di Padova, 99:219-245, 1998. 
[4] S. Amin, F. Hante, and A. Bayen. On stability of switched linear hyperbolic conservation laws with reflecting boundaries. Hybrid System: Computation and Control, Lecture Notes in Computer Science, 4981:602-605, 2008, doi:10.1007/978-3-540-78929- ${ }_{4} 4$.

[5] F. Ancona and G. Coclite. On the attainable set for Temple class systems with boundary controls. SIAM J. Control Optim., 43(6):21662190 (electronic), 2005.

[6] F. Ancona and P. Goatin. Uniqueness and stability of $\mathrm{L}^{\infty}$ solutions for Temple class systems with boundary and properties of the attainable sets. SIAM Journal on Mathematical Analysis, 34(1):28-63, 2002.

[7] F. Ancona and A. Marson. On the attainable set for scalar nonlinear conservation laws with boundary control. SIAM J. Control Optim., 36(1):290-312 (electronic), 1998.

[8] F. Ancona and A. Marson. Asymptotic stabilization of systems of conservation laws by controls acting at a single boundary point. In Control methods in PDE-dynamical systems, volume 426 of Contemp. Math., pages 1-43. Amer. Math. Soc., Providence, RI, 2007.

[9] F. AnCona and A. Marson. Existence theory by front tracking for general nonlinear hyperbolic systems. Archive for rational mechanics and analysis, 185(2):287-340, 2007.

[10] J.-P. Aubin, A. Bayen, and P. Saint-Pierre. Dirichlet problems for some Hamilton-Jacobi equations with inequality constraints. SIAM Journal on Control and Optimization, 47(5):2348-2380, 2008, doi:10.1137/060659569.

[11] C. Bardos, A.-Y. Leroux, and J.-C. Nedelec. First order quasilinear equations with boundary conditions. Communications in partial differential equations, 4(9):1017-1034, 1979.

[12] C. Bardos and O. Pironneau. Derivatives and control in the presence of shocks. Computational Fluid Dynamics Journal, 11(4):383-391, 2003.

[13] G. Bastin, B. Haut, J.-M. Coron, and B. D Andrea-Novel. Lyapunov stability analysis of networks of scalar conservation laws. Networks and Heterogeneous Media, 2(4):751-759, 2007.

[14] S. Bianchini. On the shift differentiability of the flow generated by a hyperbolic system of conservation laws. Discrete and Continuous Dynamical Systems, 6(2):329-350, 2000.

[15] S. Bianchini and A. Bressan. Vanishing viscosity solutions of nonlinear hyperbolic systems. Annals of Mathematics, pages 223-342, 2005.

[16] S. Bianchini and L. Spinolo. The boundary riemann solver coming from the real vanishing viscosity approximation. Arch. Rational Mech. Anal, page 2009 . 
[17] S. Blandin, X. Litrico, and A. Bayen. Boundary stabilization of the unviscid Burgers equation using a Lyapunov method. In Proceedings of the 49th IEEE Conference on Decision and Controls, 2010 Atlanta, Georgia, pages 1705-1712, 2010, doi:10.1109/CDC.2010.5717716.

[18] A. Bressan. The unique limit of the Glimm scheme. Archive for Rational Mechanics and Analysis, 130(3):205-230, 1995.

[19] A. Bressan. Hyperbolic systems of conservation laws: the one-dimensional Cauchy problem. Oxford University Press, Oxford, UK, 2000.

[20] A. Bressan and G. Coclite. On the boundary control of systems of conservation laws. SIAM J. Control Optim., 41(2):607-622 (electronic), 2002.

[21] A. Bressan, G. Crasta, and B. Piccoli. Well-posedness of the Cauchy problem for $n \times n$ systems of conservation laws. American Mathematical Society, Providence, RI, 2000.

[22] A. Bressan and G. Guerra. Shift-differentiabilitiy of the flow generated by a conservation law. Discrete and Continuous Dynamical Systems, 3:35$58,1997$.

[23] A. Bressan, T.-P. LiU, and T. Yang. $L^{1}$ stability estimates for $n \times n$ conservation laws. Arch. Ration. Mech. Anal., 149(1):1-22, 1999.

[24] J. Burns and S. KANG. A control problem for Burgers equation with bounded input/output. Nonlinear Dynamics, 2(4):235-262, 1991.

[25] C. Byrnes, D. Gilliam, and V. Shubov. On the global dynamics of a controlled viscous Burgers equation. Journal of Dynamical and Control Systems, 4(4):457-519, 1998.

[26] P. Christofides. Nonlinear and robust control of PDE systems: methods and applications to transport-reaction processes. Birkhäuser, Boston, 2001.

[27] J.-M. Coron, B. D'Andrea-Novel, and G. Bastin. A strict Lyapunov function for boundary control of hyperbolic systems of conservation laws. IEEE Transactions on Automatic Control, 52(1):2-11, 2007.

[28] J.-M. Coron, O. Glass, and Z. WANG. Exact boundary controllability for 1-d quasilinear hyperbolic systems with a vanishing characteristic speed. SIAM Journal on Control and Optimization, 48(5):3105-3122, 2009.

[29] C. Dafermos. Hyperbolic conservation laws in continuum physics. Springer-Verlag Berlin, Germany, 2010.

[30] J. De Halleux, C. Prieur, J.M. Coron, B. D’Andréa-Novel, and G. Bastin. Boundary feedback control in networks of open channels. Automatica, 39(8):1365-1376, 2003.

[31] M. Demetriou and I. Hussein. Estimation of spatially distributed processes using mobile spatially distributed sensor network. SIAM Journal on Control and Optimization, 48(1):266-291, 2009. 
[32] C. Donadello and A. Marson. Stability of front tracking solutions to the initial and boundary value problem for systems of conservation laws. Nonlinear Differential Equations and Applications NoDEA, 14(5-6):569$592,2007$.

[33] V. Dos Santos, G. Bastin, J.-M. Coron, and B. D'Andréa-Novel. Boundary control with integral action for hyperbolic systems of conservation laws: Stability and experiments. Automatica, 44(5):1310-1318, 2008.

[34] N. El-FArRA and P. Christofides. Coordinating feedback and switching for control of spatially distributed processes. Computers and chemical engineering, 28(1-2):111-128, 2004.

[35] L. Evans. Partial differential equations. American Mathematical Society, Providence, RI, 1998.

[36] M. Fliess, J. LÉvine, P. Martin, and P. Rouchon. Flatness and defect of non-linear systems: introductory theory and examples. International journal of control, 61(6):1327-1362, 1995.

[37] H. Frankowska. On LeFloch solutions to initial-boundary value problem for scalar conservation laws. Journal of Hyperbolic Differential Equations, $7(3): 1-41,2010$.

[38] M. Garavello and B. Piccoli. Traffic flow on networks. American Institute of Mathematical Sciences, Springfield, MO, 2006.

[39] O. GLass. On the controllability of the 1-d isentropic euler equation. $J$. Eur. Math. Soc.(JEMS), 9(3):427-486, 2007.

[40] O. GLass. On the controllability of the non-isentropic 1-d euler equation. Journal of Differential Equations, 257(3):638-719, 2014.

[41] J. GLimm. Solutions in the large for nonlinear hyperbolic systems of equations. Communication on pure and applied mathematics, 18:697-715, 1965.

[42] S. Godunov. A difference method for numerical calculation of discontinuous solutions of the equations of hydrodynamics. Matematicheskii Sbornik, 89(3):271-306, 1959.

[43] G. Gomes and R. Horowitz. Optimal freeway ramp metering using the asymmetric cell transmission model. Transportation Research Part C: Emerging Technologies, 14(4):244-262, 2006.

[44] M. Gugat and G. Leugering. Global boundary controllability of the saint-venant system for sloped canals with friction. In Annales de l'Institut Henri Poincare, volume 26, pages 257-270. Elsevier, 2009.

[45] E. Hopf. The partial differential equation $u_{t}+u u_{x}=\mu u_{x x}$. Communications on Pure and Applied Mathematics, 3(3):201-230, 1950.

[46] D. Jacquet, M. Krstic, and C. Canudas de Wit. Optimal control of scalar one-dimensional conservation laws. In 25th American Control Conference, 2006 Minneapolis, MN, USA, pages 5213-5218, 2006. 
[47] T. KobaYAshi. Adaptive regulator design of a viscous Burgers system by boundary control. IMA Journal of Mathematical Control and Information, 18(3):427, 2001.

[48] M. Krstic. On global stabilization of Burgers equation by boundary control. Systems and Control Letters, 37(3):123-141, 1999.

[49] M. Krstic and H. Deng. Stabilization of nonlinear uncertain systems. Springer New York, 1998.

[50] M. Krstic and A. Smyshlyaev. Boundary control of PDEs: A Course on Backstepping Designs. Society for Industrial and Applied Mathematics Philadelphia, PA, 2008.

[51] S. KRuzhKov. First order quasilinear equations in several independent variables. Mathematics of the USSR-Sbornik, 10(2):217-243, 1970.

[52] P. LAx. Hyperbolic systems of conservation laws II. Communications on Pure and Applied Mathematics, 10:537-566, 1957.

[53] M. Leautaud. Uniform controllability of scalar conservation laws in the vanishing viscosity limit. SIAM Journal on Control and Optimization, 50(3):1661-1699, 2012.

[54] P. LeFloch. Explicit formula for scalar non-linear conservation laws with boundary conditions. Mathematical methods in the applied sciences, 10(3):265-287, 1988.

[55] P. LEFLOCH. Hyperbolic Systems of Conservation Laws: The theory of classical and nonclassical shock waves. Lectures in Mathematics, ETH Zürich, Birkhäuser Basel, 2002.

[56] R. Leveque. Finite volume methods for hyperbolic problems. Cambridge University Press, Cambridge, UK, 2002.

[57] P. Li, R. Horowitz, L. Alvarez, J. Frankel, and M. Robertson. An AHS link layer controller for traffic flow stabilization. Transportation Research, Part C: Emerging Technologies, 5(1):11-37, 1997.

[58] M. Lighthill and G. Whitham. On kinematic waves, II: a theory of traffic flow on long crowded roads. Proceedings of the Royal Society of London, 229(1178):317-345, 1956.

[59] X. Litrico and V. Fromion. Frequency modeling of open-channel flow. Journal of Hydraulic Engineering, 130(8):806-815, 2004.

[60] X. Litrico and V. Fromion. Boundary control of hyperbolic conservation laws using a frequency domain approach. Automatica, 45(3):647-656, 2009.

[61] Tai Ping Liu. Invariants and asymptotic behavior of solutions of a conservation law. Proc. Amer. Math. Soc., 71(2):227-231, 1978.

[62] H. Ly, K. Mease, and E. Titi. Distributed and boundary control of the viscous Burgers equation. Numerical Functional Analysis and Optimization, 18(1):143-188, 1997. 
[63] O. OLeINIK. Discontinuous solutions of non-linear differential equations. Uspekhi Matematicheskikh Nauk, 12(3):3-73, 1957.

[64] F. Отто. Initial-boundary value problem for a scalar conservation law. Comptes rendus de l'Académie des sciences. Série 1, Mathématique, 322(8):729-734, 1996.

[65] V. Perrollaz. Exact controllability of scalar conservation laws with an additional control in the context of entropy solutions. SIAM Journal on Control and Optimization, 50(4):2025-2045, 2012.

[66] V. Perrollaz. Asymptotic stabilization of entropy solutions to scalar conservation laws through a stationary feedback law. In Annales de l'Institut Henri Poincare (C) Non Linear Analysis, volume 30, pages 879-915. Elsevier, 2013.

[67] C. Prieur and F. Mazenc. Iss-lyapunov functions for time-varying hyperbolic systems of balance laws. Mathematics of Control, Signals, and Systems, 24(1-2):111-134, 2012.

[68] C. Prieur, J. Winkin, and G. Bastin. Robust boundary control of systems of conservation laws. Mathematics of Control, Signals, and Systems, 20(2):173-197, 2008.

[69] P. Richards. Shock waves on the highway. Operations Research, 4(1):42$51,1956$.

[70] N. SEguin and J. VovelLe. Analysis and approximation of a scalar conservation law with a flux function with discontinuous coefficients. Mathematical Models and Methods in Applied Sciences, 13(02):221-257, 2003.

[71] D. Serre. Systems of conservation laws. Diderot, Paris, France, 1996.

[72] N. Smaoui. Boundary and distributed control of the viscous Burgers equation. Journal of Computational and Applied Mathematics, 182(1):91-104, 2005.

[73] L. Spinolo. Vanishing viscosity solutions of a $2 \times 2$ triangular hyperbolic system with dirichlet conditions on two boundaries. 2006.

[74] I. Strub and A. BAyen. Weak formulation of boundary conditions for scalar conservation laws: an application to highway traffic modelling. International Journal of Robust Nonlinear Control, 16(16):733-748, 2006, doi: 10.1002/rnc.1099.

[75] B. Temple. Systems of conversation laws with invariant submanifolds. Transactions of the American Mathematical Society, 280(2):781-795, 1983.

[76] R. Vazquez, J.-M. Coron, M. Krstic, and G. Bastin. Local exponential $h^{2}$ stabilization of a $2 \times 2$ quasilinear hyperbolic system using backstepping. In Decision and Control and European Control Conference (CDC-ECC), 2011 50th IEEE Conference on, pages 1329-1334. IEEE, 2011.

[77] D. Work, S. Blandin, O.-P. Tossavainen, B. Piccoli, and A. Bayen. A traffic model for velocity data assimilation. Applied Mathematics Research eXpress, 1:1-35, 2010, doi:10.1093/amrx/abq002. 